\title{
Nanohydroxyapatite, Nanosilicate-Reinforced Injectable, and Biomimetic Gelatin-Methacryloyl Hydrogel for Bone Tissue Engineering
}

\author{
Zhe Shi (i)* \\ Qiang Zhong* \\ Yuhang Chen \\ Jian Gao \\ Xin Pan \\ Qiang Lian \\ Rong Chen \\ Pinkai Wang \\ Jian Wang \\ Zhanjun Shi \\ Hao Cheng
}

Department of Orthopedics, Nanfang Hospital, Southern Medical University,

Guangzhou, Guangdong Province,

5I05I5, People's Republic of China

*These authors contributed equally to this work
Correspondence: Zhanjun Shi; Hao Cheng Tel +862062787924

Email shizhanjun I I @gmail.com;

haocool317@gmail.com
Purpose: Given that autologous bone graft for bone defects is limited by insufficient supply and morbidity at the donor site, developing biomimetic graft materials as an alternative has gained consistent attention. However, obstacles in designing bone-mimetic materials that could integrate the biomimetic nature of the bone extracellular matrix, osteogenic cells, and osteoinductive ingredients with a fast and convenient strategy still exist.

Methods: This study designed and fabricated a mesenchymal stem cell (MSC)-laden, nanohydroxyapatite (HAP), and nanosilicate (SN)-loaded bone mimetic and injectable gelatin-methacryloyl hydrogel (GelMA-HAP-SN) system for bone tissue engineering, and systemically investigated the osteogenic capacity of GelMA-HAP-SN in vitro and in vivo.

Results: Introducing HAP enhanced the compositional similarity to the natural bone extracellular matrix, and SN loading endowed the hydrogel with injectable and osteogenic ability. As a result, the GelMA-HAP-SN hydrogel demonstrated an increase in cellular viability, proliferation, and spreading behavior. The GelMA-HAP-SN hydrogel also amplified the embedded MSCs' osteogenic biomarkers' expression and matrix mineralization. Furthermore, the MSC-encapsulated GelMA-HAP-SN hydrogel was injected into rats' critical-sized calvaria defect, and micro-CT and histomorphometry staining results further confirmed its excellent bone regeneration ability.

Conclusion: These MSC-loaded GelMA-HAP-SN hydrogels are potential graft materials for bone defect treatment.

Keywords: GelMA, injectable, biomimetic, nanohydroxyapatite, nanosilicate, bone regeneration

\section{Introduction}

Managing bone defects remains challenging for patients and surgeons, ${ }^{1}$ as inappropriate treatment causes significant disability in patients. ${ }^{2}$ Autologous bone graft is the gold standard for bone defect treatment, as the transplanted bone tissue can quickly fit and grow into the native bone defect area. ${ }^{3-5}$ However, the limitation on supply and procurement morbidity at the donor site restricts its use. ${ }^{6}$ Thus, various bone graft materials from inorganics to organics, as an alternative, have been widely applied, ${ }^{7-10}$ among which synthetic and metallic substitutes are widely used with the advantage of unlimited supply, easy sterilization and storage, and excellent mechanical strength and biocompatibility. ${ }^{7-9}$ However, the lack of osteoinductivity of these substitutes delays bone union, and the mismatch of mechanical strength to natural bone tissue impedes bone remodeling, causing 
concerns of implant loosening. Also, the aforementioned bone substitutes rarely possess inherent bone regeneration ability because of their incapability of carrying stem cells. To overcome these deficiencies and adapt to the commonly irregular-shape defects, injectable biomaterials for bone tissue engineering have been attracting considerable attention $^{11-13}$ for filling the irregular defect with a minimally invasive procedure. However, most fabrication strategies for this injectable biomaterial are complicated and time-consuming. ${ }^{12,13}$ Thus, to guarantee efficient bone regeneration, injectable biomaterials with quick and convenient strategy, which could efficiently integrate chemical and structural similarity to natural bone, osteogenic cells, and osteoinductive ingredients, are highly desired.

Recently, hydrogels as biologically active scaffolds have gained considerable interest in bone tissue engineering, ${ }^{11}$ as the prepolymer used could highly mimic the physicochemical properties of the natural bone extracellular matrix (ECM) ${ }^{14-18}$ Besides, hydrogels can provide a three-dimensional (3D) culture platform for cell encapsulation, proliferation, differentiation, and bioactive ingredient incorporation and release. ${ }^{19-21}$ Moreover, its tunable mechanical properties by adjusting the crosslinking parameters provide the advantage of meeting the various stiffness demands of the specific tissue microenvironment. For instance, gelatin-based hydrogel has been widely used for biomedical applications. Gelatin is a denatured collagen obtained from various animal body parts while retaining cell-binding sequence Arg-Gly-Asp (RGD) and matrix metalloproteinase (MMP)-sensitive degradation motifs. ${ }^{22}$ After the amino-side groups in gelatin conjugated to the methacrylate groups, methacryloylated gelatin (GelMA) becomes light-polymerizable and could be cross-linked into a hydrogel form with ultraviolet (UV) radiation. Thus, GelMA demonstrated its versatility as a platform with appropriate stiffness for various tissue engineering applications, including enveloping bone and cartilage regeneration, myocardial repairing, and revascularization. ${ }^{23}$ Additionally, composite hydrogel systems for biomimetic bone graft materials can be fabricated by intruding ceramic nanoparticles, such as nanohydroxyapatite (HAP) and $\mathrm{CaCO}_{3}$, into GelMA. ${ }^{21,24} \mathrm{HAP}$, a major inorganic component of natural bones and teeth, has been popularly used as additives during the fabrication of bone-mimetic substitutes and bone tissue engineering polymer. ${ }^{25-27}$ After adding HAP, the hydrogel composites show significant improvement in mechanical properties, structural and compositional similarities to natural bone ECM, and promoted biocompatibility by preserving cellular viability and enhancing cellular proliferation. ${ }^{25-27}$ However, as the osteogenic capability of HAP is limited, the osteoinductive capability of pure GelMA-HAP hydrogel could not be guaranteed.

Nanosilicate (SN) [Laponite: $\mathrm{Na}_{0.7}^{+}\left[\left(\mathrm{Si}_{8} \mathrm{Mg}_{5.5}: \mathrm{Li}_{0.3}\right.\right.$ $\left.\left.\mathrm{O}_{20}(\mathrm{OH})_{4}\right]_{0.7}^{-}\right)$, a charged synthetic bioactive silicate nanoplatelet with an average diameter of $20-30 \mathrm{~nm}$ and thickness of $1 \mathrm{~nm}$, has recently gained considerable attention due to its superior osteoinductive potential. ${ }^{28,29}$ Magnesium ions released from dissociated SN promoted initial cellular adhesion to the surface of biomaterials by mediating adhesion proteins of integrin family expression. ${ }^{30}$ Also, degradation products of $\mathrm{SN}$, such as orthosilicic acid $\left(\mathrm{Si}(\mathrm{OH})_{4}\right)$ and lithium, further play an important role in osteogenesis. ${ }^{31,32}$ Orthosilicic acid promotes the synthesis of the vital bone ECM component, namely, the collagen type I. ${ }^{32,33}$ However, lithium further boosts the osteogenesis of $\mathrm{SN}$ by regulating the expression of runt-related transcription factor-2 (RUNX2), the major osteogenic regulator responsible for coordinating the expression of bone-specific genes. ${ }^{31,34,35}$ Altogether, SN can induce the osteogenic differentiation of mesenchymal stem cells (MSCs) efficiently without any auxiliary exogenous osteoinductive factors. Given the osteogenesis brought by $\mathrm{SN}$ as stated above, the nanoplatelet with HAP contributes to the osteogenic microenvironment. Apart from the osteogenic ability, synthetic SN could increase viscosity and fabricate secondary networks of the hydrogel due to its electrostatic interactions and van der Waals forces. ${ }^{36,37}$ The nonbonding complexation will decrease the cross-linking time of UV radiation for hydrogel polymerization, which is beneficial for preserving cell viability. In contrast, shear-thinning characteristic of $\mathrm{SN}$, a phenomenon in which viscosity decreases with increasing shear stress, also boosts the injectable potential of GelMA-HAP hydrogel, making the fabrication and application of injectable hydrogel more convenient than other injectable strategies. ${ }^{12,13}$ After cell encapsulation, the injectable GelMA-HAP-SN hydrogel can fill irregular defects and promote in situ bone regeneration by transporting the osteoprogenitor cells with the osteogenic microenvironment established by osteoinductive ingredients in a minimally invasive way.

This study developed a biomimetic, osteogenic, injectable, and cell-loaded hydrogel with a fast and clinically available strategy; HAP, SN, and MSCs were introduced 
into GelMA hydrogel. The introduction of HAP endowed the GelMA hydrogel with chemical and structural similarity to natural bone. Additionally, adding SN to the GelMA-HAP hydrogel could further endow the nanocomposite with the desired osteoinductive property and injectability. To verify our hypothesis, we began this study by optimizing the concentration of loaded HAP and SN in the GelMA hydrogel; then, we investigated the cellular preservation, proliferation, and differentiation of MSCs encapsulated in the hydrogel. Furthermore, we injected the MSC-loaded biomimetic nanocomposite hydrogel and cross-linked, in situ, in critical-sized calvaria defect built on Sprague-Dawley (SD) rats. Finally, the osteogenic ability of the MSC-loaded GelMA-HAP-SN hydrogel was evaluated systematically. Altogether, it was expected that this injectable and bone-mimetic nanocomposite hydrogel fabricated with a simple and applicable strategy could address the challenge of bone defect treatment.

\section{Materials and Methods Materials}

Gelatin from porcine skin (gel strength 300, Type A), methacrylic anhydride (94\%), BSA (98\%), Irgacure 2959 (98\%), Alizarin Red S, and hydroxyapatite nanoparticles $(<200 \mathrm{~nm}$, 12167-74-7) were acquired from SigmaAldrich (St. Louis, MO, USA). SN XLG was purchased from BYK Additives and Instruments (Wesel, Germany). Live/dead viability/cytotoxicity kit, $\beta$-glycerol phosphate 173 (99\%), L-ascorbic acid (99\%), Alexa Fluor 594/488 -phalloidin, 4',6-diamidino-2-phenylindole (DAPI), $\alpha$ minimum essential medium ( $\alpha$-MEM), trypsin-ethylenediaminetetraacetic acid (trypsin-EDTA), L-glutamine, and antibiotics (penicillin/streptomycin) were acquired from Thermo Fisher Scientific (Waltham, MA, USA). Antialkaline phosphatase (ALP) antibody (sc-271431) was acquired from Santa Cruz Biotechnology (Dallas, TX, USA). Anti-OCN antibody (MABD123), Anti-OPN antibody (AB1870), and Anti-RUNX2 antibody (05-1478) were provided by Millipore (Burlington, MA, USA).

\section{Synthesis of GelMA Macromonomers}

Based on established protocols, ${ }^{23}$ GelMA macromonomers were synthesized using gelatin from porcine skin and methacrylic anhydride. Briefly, 5-g gelatin was first completely dissolved in $50-\mathrm{mL}$ phosphate-buffered saline (PBS, $\mathrm{pH}=7.4,50^{\circ} \mathrm{C}$ ) under magnetic stirring. Next, 0.625 -g methacrylic anhydride (MA) was transferred into the working solution to react with gelatin at $50^{\circ} \mathrm{C}$ and incubated for $1 \mathrm{~h}$. The reaction was terminated by dilution by adding $200-\mathrm{mL}$ PBS. The diluted solution was then transferred to the dialysis membrane $(10 \mathrm{kDa}$ cut-off dialysis membrane, $\mathrm{pH} \sim 7-7.4,40^{\circ} \mathrm{C}$ ) in the dark and dialyzed for one week to remove unreacted MA. Finally, the ultimate solution was lyophilized $\left(-80^{\circ} \mathrm{C}\right.$ overnight $)$ to obtain GelMA macromonomers in the form of white foam.

\section{Fabrication of GelMA-Based Hydrogel}

To prepare the GelMA-based hydrogel samples, GelMA macromonomer $(10 \% \mathrm{w} / \mathrm{v})$ mentioned above with Irgacure $2959(0.5 \% \mathrm{w} / \mathrm{v})$ as the photoinitiator was fully dissolved in PBS. Besides, HAP and SN were separately (GelMAHAP or GelMA-SN) or jointly (GelMA-HAP-SN) blended into the prepolymer. Then, after cross-linking (30-s UV radiation, $6.9 \mathrm{~mW} / \mathrm{cm}^{2}, 360-480 \mathrm{~nm}$ ) in customized polydimethylsiloxane (PDMS) mold, the GelMA-based hydrogel samples were obtained.

HAP and SN of different concentrations were separately added to the hydrogel samples to define the optimal concentration of the nanoparticles. CCK-8 assay was conducted to clarify the optimal concentration.

\section{Characterization of GelMA-Based Hydrogel}

The nano HAP and SN morphology, GelMA hydrogel injectability, porous structure, mechanic structure, swelling ratio, and degradation behavior were analyzed.

\section{Viscosity and Injectability Analysis}

The viscosity of GelMA-SN rheology was analyzed by stress recovery tests. ElastoSensTM Bio2 (Rheolution Instruments, Montreal, QC, Canada) was used to test the hydrogel samples (uncross-linked), loaded with different SN weights $(0 \%, 1 \%$, and $2 \%)$ in a shear rate range of $0.1-100$ $\mathrm{S}^{-1}$ to obtain the shear stress at room temperature. The hydrogel (uncross-linked) storage modulus $\left(\mathrm{G}^{\prime}\right)$ at $37^{\circ} \mathrm{C}$ was also evaluated using the ElastoSensTM Bio2. The hydrogel was subjected to sequential high $(100 \%, 10 \mathrm{~min})$ and low $(1 \%, 10 \mathrm{~min})$ strain rates at $1 \mathrm{~Hz}$. Injectability was assessed using Instron 5940 (Instron, Norwood, MA, USA) with 19-, 21-, 23-, and 25-needle at room temperature. In detail, the injection force was analyzed by injecting GelMASN hydrogel (2\%) from a 1-mL syringe with 1.5 -inch needles at a flow rate of $2 \mathrm{~mL} / \mathrm{h}$. Power-law model fit data were obtained using the rheometer (Rheolution Instruments, Montreal, QC, Canada) at room temperature. A power-law equation was then used to determine the power-law index 
(n), consistency index $(\mathrm{k})$, and the correlation coefficient (Corr. Coeff) values. The following power-law equation used is presented below:

$\sigma=\mathrm{k} \ddot{\mathrm{Y}} \mathrm{n},(\sigma=$ Shear stress; $\mathrm{k}=$ Consistency; $\ddot{\mathrm{Y}}=$ Shear rate; $\mathrm{n}=$ Power-law index).

\section{Scanning Electron Microscopy (SEM) and Transmission Electron Microscopy (TEM) Observation}

The nano HAP and SN morphologies were observed by TEM (JEOL JEM-2100Plus), and lyophilized hydrogel samples were scanned using a field emission SEM (Zeiss Ultra 55) to analyze the internal microstructure. The average pore size of the lyophilized hydrogel samples was obtained by measuring the SEM results using ImageJ software (v.1.44).

Fourier-Transform Infrared Spectroscopy (FT-IR) and X-Ray Diffraction (XRD) Analysis

To analyze the chemical functional groups of the hydrogel, powdered samples were first obtained by grinding the lyophilized foam, then mixed with potassium bromide, and finally pressed into a tablet. Then, the processed samples were analyzed using FT-IR (Bruker Vertex 70) at room temperature.

To verify the crystal structure of nanoparticles in the hydrogel samples, XRD was conducted between 10 and 80 degrees in a diffractometer (Bruker D8). As a result, the crystallinity of HAP and SN was estimated according to the formula below:

Crystallinity $=($ Area of crystalline peaks/Area of all peaks) $\times 100 \%$

\section{Mechanical Analysis}

The cylindrical hydrogel samples ( $8 \mathrm{~mm}$ wide, $2 \mathrm{~mm}$ high) were prepared as mentioned before, and the above hydrogel samples were transferred to an Instron 5542 mechanical tester and compressed for $30 \mathrm{~s}$ at a speed of $1 \mathrm{~mm} / \mathrm{min}$ to conduct mechanical analysis. The first $0-10 \%$ linear region of the stress-strain curve was adopted to calculate Young's modulus.

\section{Degradation and Swelling Analysis}

For the degradation assay, cylindrical hydrogel samples were prepared as before. Freeze-dried hydrogel samples were weighed, and the results were recorded as the initial mass. After immersion in PBS at $37^{\circ} \mathrm{C}$, the hydrogel samples were extracted, lyophilized, and weighed at preset time points. The degradation profile was then calculated.
For the swelling test, cylindrical hydrogel samples were incubated in PBS for $1 \mathrm{~h}$ to reach the equilibrium state. First, the mass of the swollen hydrogel was weighed as $\mathrm{M}(\mathrm{s})$. Then, the hydrogel was lyophilized and weighted as $\mathrm{M}(\mathrm{l})$. Finally, the swelling ratio was calculated based on the following equation:

Swelling ratio $=[\mathrm{M}(\mathrm{s})-\mathrm{M}(\mathrm{l})] / \mathrm{M}(\mathrm{l})$

\section{Cell Isolation and Encapsulation in the Nanocomposite Hydrogel Scaffold}

Rat bone marrow MSCs were isolated according to a previous study. ${ }^{38}$ Briefly, six-week male rats' femur and tibia were harvested, and the epiphyseal ends were removed. Bone marrow was flushed out by injecting a culture medium with a $5-\mathrm{mL}$ syringe with a 25 $\mathrm{G}$ needle. The acquired bone marrow suspension was filtered with a 200-mesh sieve and centrifuged. After the resuspension of the pellets in the growth medium, the abstained MSCs were evenly transferred into T25 flasks, followed by incubation at $37^{\circ} \mathrm{C}$ with $5 \% \mathrm{CO}_{2}$. The first cell medium refreshment was performed after $48 \mathrm{~h}$, and a subsequent renewal was performed every $3 \mathrm{~d}$ in the following week until $80-90 \%$ cell confluence was observed. MSCs were cultured until passage three and then used in the following experiments.

To encapsulate MSCs in the hydrogel samples, $1 \times 10^{6}$ cells $/ \mathrm{mL}$ of MSCs was added to the GelMA prepolymer solution (with or without nanoparticles) and photocrosslinked by UV exposure for $30 \mathrm{~s}$, which is the optimized time based on our previous experience. MSCs encapsulated in the samples were then nourished with osteoconductive medium, Dulbecco's modified Eagle's medium containing fetal bovine serum (FBS, 10\%), penicillin-streptomycin (1\%), L-ascorbic acid (50 $\mu \mathrm{g} / \mathrm{mL})$, and $\beta$-glycerol phosphate $(10 \mathrm{mM} / \mathrm{mL})$. Only MSCs within passage five were allowed for the experiments. The medium was refreshed every $2-3 \mathrm{~d}$.

\section{Cell Viability, Proliferation, and Spreading Analysis in the Nanocomposite Hydrogel Scaffold}

To evaluate the cellular viability of the encapsulated cell in the hydrogel sample on days 1,3 , and 7 , calcein acetoxymethyl and ethidium homodimer-1 in the live/dead assay kit were applied to label live and dead cells, respectively. After staining, the results were recorded using an inverted fluorescence microscope (Nikon TE 2000-U), and the stained cells were calculated using ImageJ software 
(v.1.44). Cell viability was obtained by comparing the quantity of live cells to the total amount of cells.

Additionally, to compare the spreading behavior of cells cultured in 3D hydrogel scaffolds on days 3 and 7, nuclei and actin in the encapsulated MSCs were labeled by 4',6-diamidino-2-phenylindole (DAPI) and Alexa Fluor 594 phalloidin staining, respectively. First, samples were fixed with $4 \%(\mathrm{v} / \mathrm{v})$ paraformaldehyde, followed by cell membrane permeabilization with a $0.1 \%$ (w/v) Triton X-100 solution. Then, the samples were cultured in $1 \%$ (w/v) bovine serum albumin (BSA, 98\%, Sigma-Aldrich) solution to block non-specific binding sites. Finally, hydrogel samples were immersed in Alexa Fluor 594 phalloidin solution for $90 \mathrm{~min}$ and DAPI solution for $10 \mathrm{~min}$ in that order. The staining results were recorded using a confocal microscope (Zeiss LSM 980), and the cell-spreading area was calculated using ImageJ software (v.1.44).

\section{Osteogenic Biomarkers Expression of the MSCs Encapsulated in the Nanocomposite Hydrogel}

To assess the osteogenic biomarker expression level of the cells loaded in the hydrogel samples, we first used RTPCR to analyze gene expression related to osteogenesis, including the four typical genes of ALP, osteocalcin $(\mathrm{OCN})$, osteopontin $(\mathrm{OPN})$, and runt-related transcription factor 2 (RUNX2). The primers used in our study are listed in Table S1. On days 14 and 28, cell-encapsulated samples were taken out of the osteoconductive medium and lysed using Trizol (Invitrogen). Then, quantitative reverse transcription-polymerase chain reaction (qRTPCR) was performed using a Bio-rad MyiQ2 thermocycler with the SYBRR Green Supermix (Biorad).

We used ALP, OCN, OPN, and RUNX2 antibodies to label the target proteins to further evaluate protein expression levels associated with osteogenic differentiation in the hydrogel. Hydrogel samples were first fixed with paraformaldehyde (4\%) and permeabilized with Triton X-100 solution. Then, $4 \%$ normal goat serum was used to block the nonspecific antigens, primary and secondary antibody solutions were used to culture the hydrogel samples for 2 and 1 $\mathrm{h}$, at $37^{\circ} \mathrm{C}$ with $5 \% \mathrm{CO}_{2}$. Finally, actin and cellular nuclei were marked with Alexa Fluor 594/488 phalloidin and DAPI, respectively. The staining results were observed and recorded using a confocal microscope, and the target proteinlabeled area was quantified using ImageJ software (v.1.44).

\section{Matrix Mineralization Analysis}

The effects of HAP and/or SN nanoparticles on mineralized extracellular matrix production in two-dimensional (2D) and 3D conditions were determined by Alizarin Red staining. After 14- and 28-days of culture, 2\% (w/v) Alizarin Red S ( $\mathrm{pH}=4.2)$ was added to the cells and samples at room temperature for $10 \mathrm{~min}$. For quantification, $10 \%(\mathrm{v} / \mathrm{v})$ acetic acid was applied for a 30-min acidification of the stained samples. Subsequently, the acetic acid was neutralized with $10 \%$ ammonium hydroxide, and the resultant supernatant was measured using a microplate reader at a wavelength of $405 \mathrm{~nm}$.

\section{Surgical Procedure of Rat Calvaria Defect Model and Nanocomposite Hydrogel Transplantation}

SD rats used in this study were provided by the Laboratory Animal Centre of Nanfang Hospital of Southern Medical University. All experimental procedures were approved by the Animal Experimental Ethics Committee of Nanfang Hospital of Southern Medical University (NFYY-2018-81), and the animals were kept following the guidelines of the National Institutes of Health guide for the care and use of Laboratory animals (NIH Publications No. 8023, revised 1978). Four groups of ten male SD rats $(\sim 120 \mathrm{~g}, 40$ rats in all) were equally divided, including a control GelMA hydrogel group, a GelMA-HAP hydrogel group, a GelMA-SN hydrogel group, and a GelMA-HAP-SN hydrogel group. All rats were anesthetized thoroughly by intraperitoneal injection of pentobarbital sodium. The surgical area was shaved using electric clippers and disinfected with anil iodine. A $1.5-\mathrm{cm}$ median incision was created for calvarium exposure, and the covering periosteum was retracted. A criticalsized calvarial defect was made using a trephine with $8-\mathrm{mm}$ diameter. Prefabricated cylindrical hydrogel samples $(8 \mathrm{~mm}$ in diameter, $2 \mathrm{~mm}$ in height) were transplanted into the defect area. The periosteum and skin were closed and disinfected.

\section{Bone Regeneration Analysed by Micro CT and Histomorphometry Staining}

At eight-week post-surgery, the rats were terminated with an overdose of pentobarbital sodium. The rats' skulls were gathered and scanned by micro-CT to assess new bone regeneration. Furthermore, the histomorphometry characteristics of the regenerated bone structure were analyzed by hematoxylin-eosin ( $\mathrm{H}$ and $\mathrm{E}$ ) staining and GoldnerMasson trichrome staining. $\mathrm{H}$ and $\mathrm{E}$ and Goldner- 
Masson trichrome staining kits were used following the manufacturer's instructions. Then, the staining results were ascertained under the microscope and investigated using ImageJ software (v.1.44). The damaged width defect area was documented as $\mathrm{D}(\mathrm{a})$; the healing width was calculated as $8-\mathrm{D}(\mathrm{a})$. Finally, the bone-healing percentage was computed using the following equation:

Bone healing percentage $=[8-\mathrm{D}(\mathrm{a})] / 8 \times 100 \%$

For the quantification of new bone and osteoid formation from the Goldner-Masson trichrome staining outcomes, the regions of interest (ROI) manager features were proven by ImageJ software (v.1.44). Then, the analyze-set measurement step was applied to select the parameters.

\section{Statistical Analysis}

All experiments conducted in this study were conducted in triplicate. Experimental results were compared by one-way analysis of variance followed by Bonferroni's post hoc analysis (GraphPad Prism v.6.0 software) to detect statistical significance at $* p<0.05$ and $* * p<0.01$.

\section{Results}

\section{Characterization of Nanocomposite GelMA-Based Hydrogels}

The synthetic process and the acquired macromonomer of GelMA hydrogel are displayed in Figure 1A and Figure S1, and the assemblage process of nanocomposite hydrogels, including HAP and SN, was approached step by step. Crosslinked GelMA hydrogel samples are illustrated in Figure 1B. The methacrylation degree was contained by supplemental anhydride and reaction time. Based on a previous study, the degree of GelMA hydrogel methacrylation was $\sim 53.8 \%$ when $1.25 \% \mathrm{w} / \mathrm{v}$ MA reacted with $10 \%$ gelatin during the synthetic process. ${ }^{22}$ Additionally, HAP and SN exhibited rhombohedral (size $100 \mathrm{~nm}$ ) and platelet-like shapes (size $30 \mathrm{~nm}$ ), respectively (Figure 1C (i) and (ii)). The nanocomposite hydrogel microstructure revealed an interconnected porous network, and HAP and SN were homogeneously dispersed in the hydrogel network (Figure 1C (iii) and (iv)). The average pore size was $200 \mu \mathrm{m}$ for the GelMA hydrogel, and auxiliary HAP did not change the parameter significantly. Nonetheless, the inclusion of SN reduced the pore size to $\sim 150 \mu \mathrm{m}$ (Figure S2). The lyophilized pore size was smaller than the pore size in the wet state, which was possibly due to dehydration and shrinking during freeze-drying (Figure S4).
The injection force of $2 \%$ GelMA-SN was elevated from $\sim 2.4$ to $6.9 \mathrm{~N}$ when the needle size was decreased from $19 \mathrm{G}$ to $25 \mathrm{G}$, respectively (Figure 1D). The addition of HAP to GelMA-SN did not affect the injectability (Figure S1). After the loading of SN (1 and $2 \mathrm{wt} \%$ ), the GelMA's (uncross-linked) viscosity increased from $\sim 1$ to 410 poise, demonstrating exemplary shear-thinning properties (Figure 1E). Furthermore, the incorporation of $\mathrm{SN}$ enhanced the prepolymers shear recovery capability (Figure $1 \mathrm{~F}$ ). In addition, the integration of $1 \%$ GelMA-SN increased the storage modulus to $\sim 53 \mathrm{~Pa}$, whereas $2 \%$ GelMA-SN created a storage modulus of $\sim 230 \mathrm{~Pa}$. Following exposure to high strain (100\%), the $2 \%$ GelMA-SN prepolymer was retrieved from $\sim 70 \%$ to $80 \%$ of the initial storage modulus. The power-law (n) and consistency indexes (k) were $\sim 0.25$ and $\sim 25$ for the $1 \%$ GelMA-SN hydrogel and $\sim 0.22$ and $\sim 57$ for the $2 \%$ GelMASN hydrogel, respectively (Table S2). FT-IR results and analysis supported that no new enhanced or shifted peak of the amide functional groups appeared after nanoparticle loading, implying that there was no chemical bond production between the nanoparticles and GelMA backbone (Figure 1G). Besides, the XRD results suggested that the relative crystallinity of HAP and SN were $97.63 \%$ and $19.86 \%$, respectively.

The Young's modulus was positively associated with loaded HAP and SN concentration in the nanocomposite hydrogel. Specifically, when the concentration of nanoparticles of HAP increased from 1 to $1000 \mu \mathrm{g} / \mathrm{mL}$, the stiffness of the GelMA-HAP hydrogel was amplified from $\sim 15$ $\sim 39 \mathrm{kPa}$ (Figure S3). The modulus of 1\% GelMA-SN and $4 \%$ GelMA-SN was $\sim 16 \mathrm{kPa}$ and $\sim 33 \mathrm{kPa}$ (Figure S3), respectively. To construct GelMA-based hydrogel samples with preset stiffness, the integration of HAP and SN significantly reduced the UV radiation time (Figure S3). The hydrogel samples presented a similar swelling ratio and degradation profile (Figure 1I and Figure 1J); also, HAP and SN incorporation did not modify the hydrogel's swelling ratio and degradation speed (Figure 1G).

\section{Growth and Spreading of MSCs Embedded in HAP-SN Nanocomposite GelMA Hydrogel}

To resolve the optimal nanoparticle concentration for MSC proliferation in a 3D hydrogel, the HAP concentration supplemented into the hydrogel ranged from 1 to $1000 \mu \mathrm{g} / \mathrm{mL}$. In contrast, SN was introduced into the hydrogel from $1 \%$ to $4 \%$ $\mathrm{w} / \mathrm{v}$. The viability and proliferation of encapsulated cells were analyzed and recorded (Figure 2A and B). The most 
A

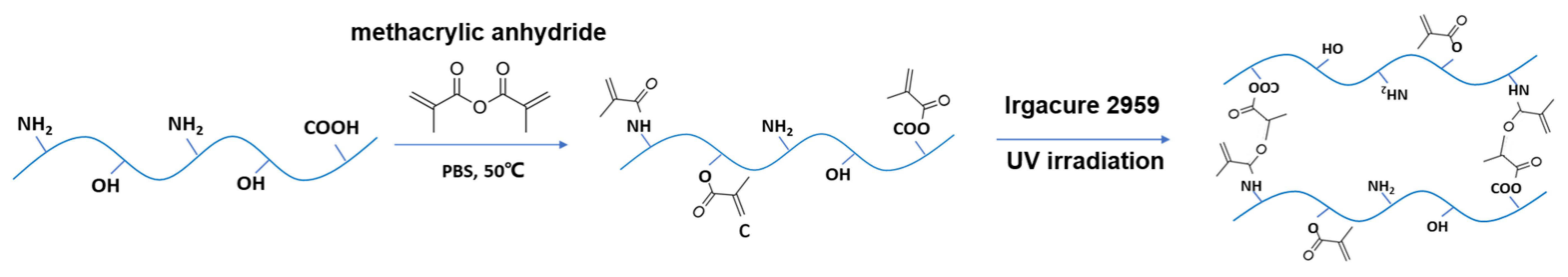

B
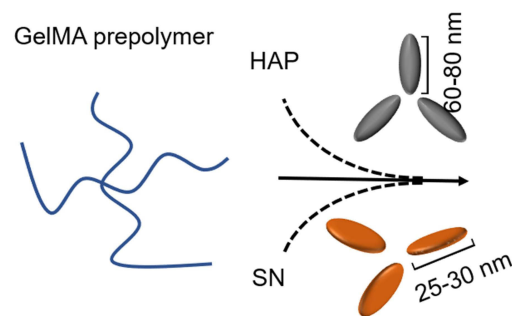

GeIMA-HAP-SN

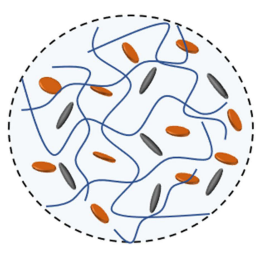

UV crosslinking

C
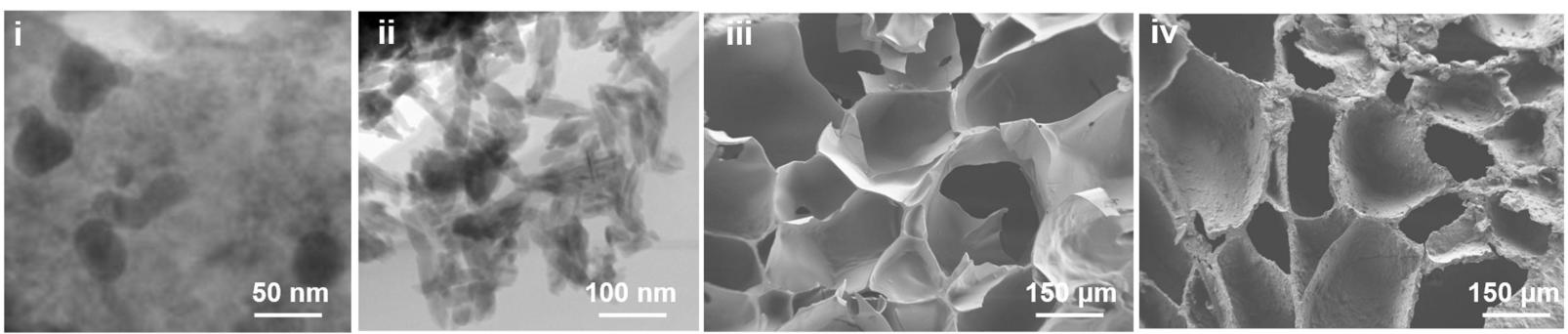

D

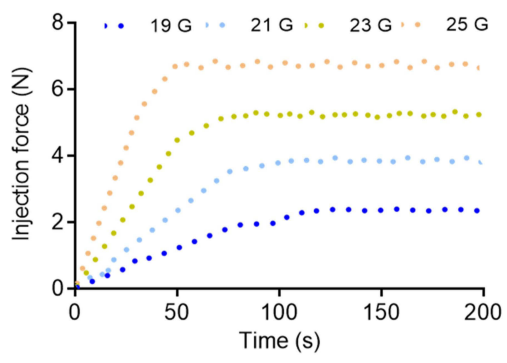

G

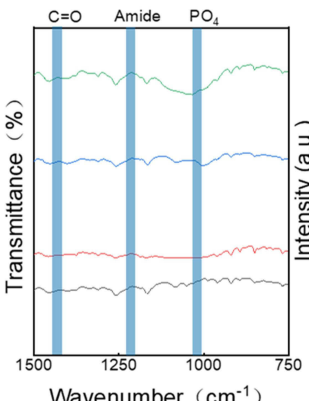

Wavenumber $\left(\mathrm{cm}^{-1}\right)$
E

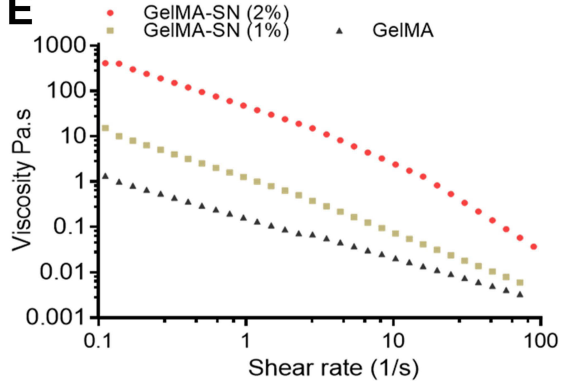

H
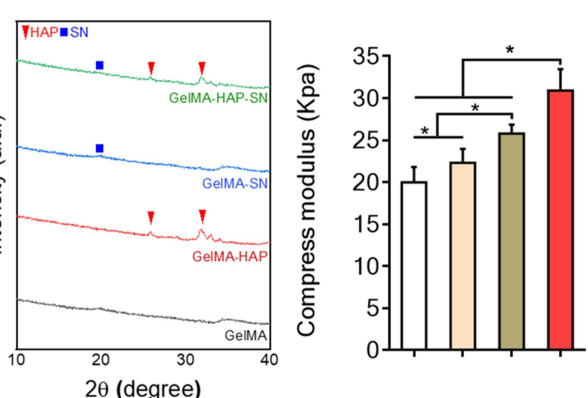

I

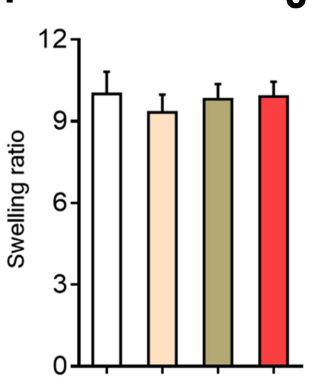

J

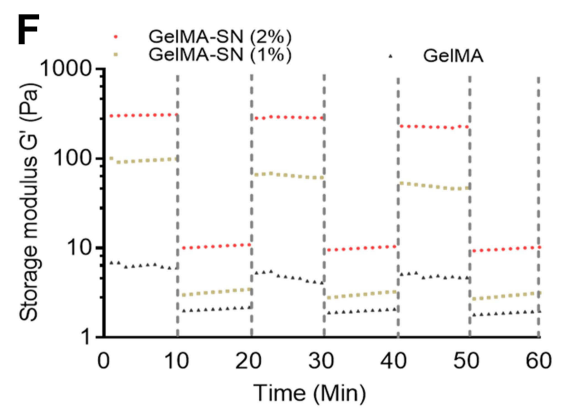

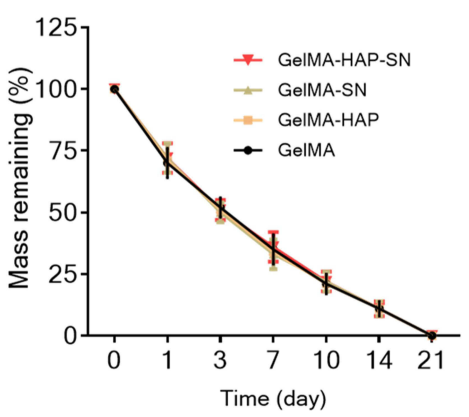

Figure I Fabrication and characterization assay of GeIMA-HAP-SN hydrogel. (A) Chemical synthesis of the GelMA from gelatin and methacrylic anhydride and UV crosslinking structure of the GelMA. (B) Fabrication of GelMA-HAP-SN hydrogel. (C) Electron microscopy of the nanoparticles and the hydrogel. i-ii TEM observation of SN and HAP; iii-iv SEM observation of GelMA with and without the nanoparticles. (D) GelMA-HAP-SN hydrogel (uncross-linked) injectability demonstrated using a syringe with various needle sizes at room temperature. (E-F) Viscosity and storage modulus assay of SN-loaded GelMA hydrogel with various SN concentrations. (G) Chemical composition assay of GelMA, GeIMA-HAP, GelMA-SN, and GelMA-HAP-SN hydrogel using Fourier-transform infrared spectrometer X-ray diffraction. (H-I) Calculated swelling rate and compression modulus of GelMA-based hydrogel after UV cross-linking. (J) Degradation rate of GelMA, GelMA-HAP, GelMA-SN, and GelMA-HAP-SN hydrogel after UV cross-linking. $* P<0.05$ indicates statistical difference. 
A

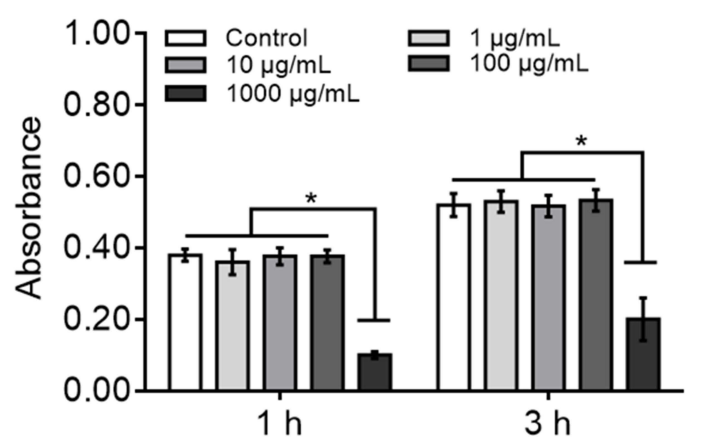

C

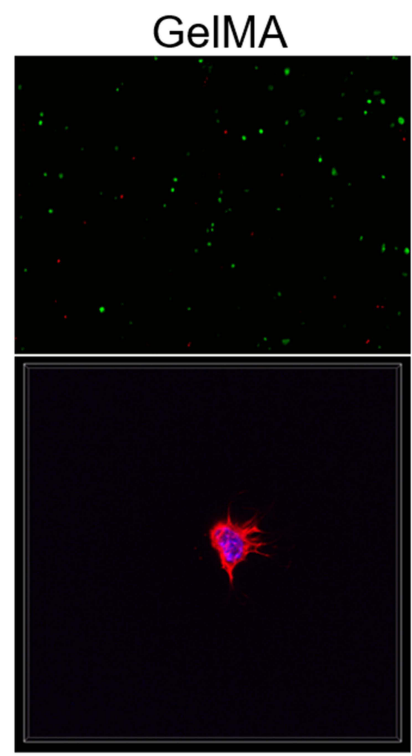

D

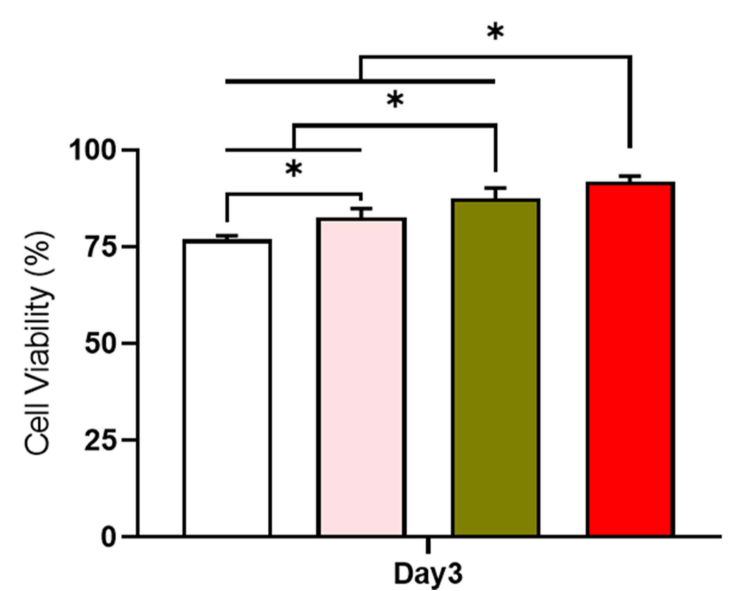

GelMA-HAP
B
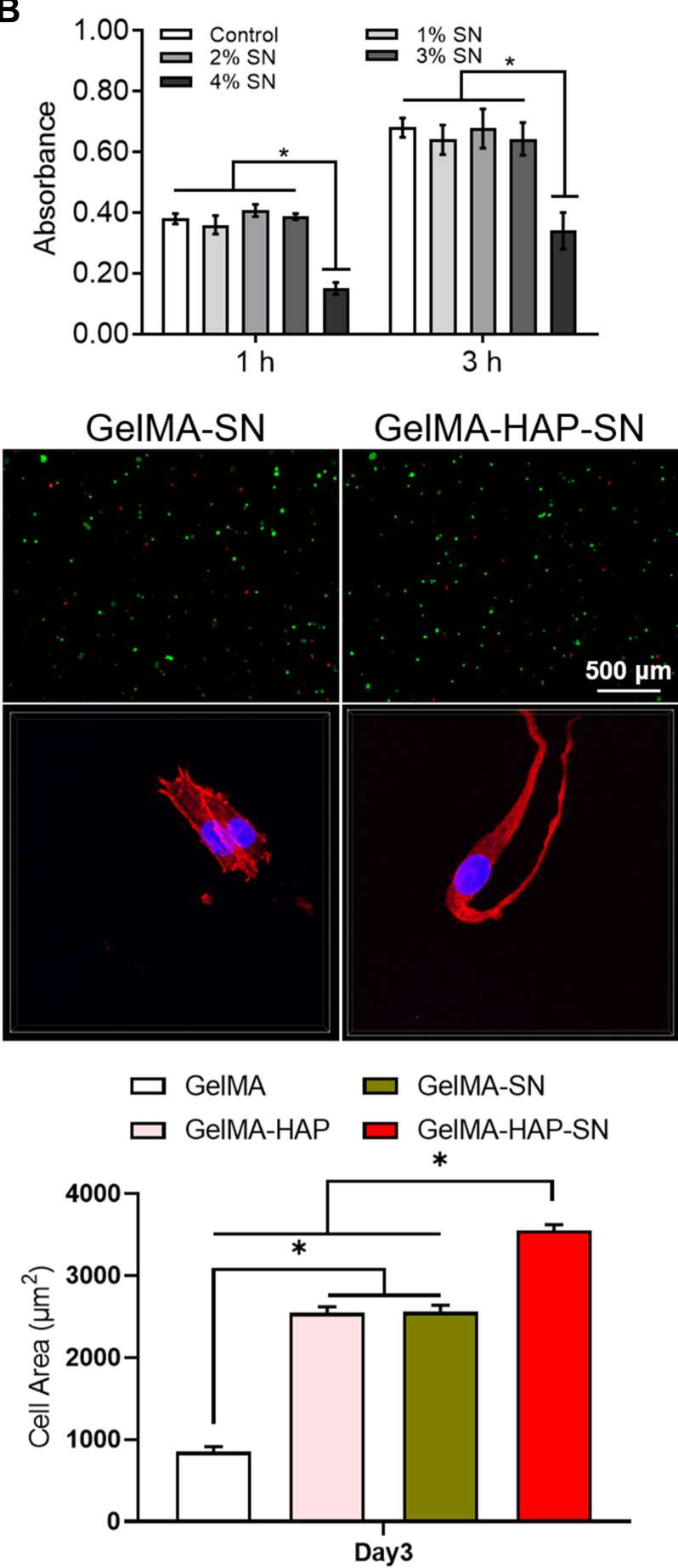

Figure 2 Growth and expansion of MSCs embedded in HAP-SN nanocomposite hydrogel. (A-B) Absorbance rate by CCK-8 assay of the GelMA-based hydrogels with various HAP or SN concentrations, respectively. (C) Cell viability labeled by live-dead assay and spreading behavior by DAPI (blue)- phalloidin (red) on Day 3 in the GelMAbased hydrogels demonstrated. (D) Quantification of cell viability and spreading area by Image. $* P<0.05$ indicates statistical difference.

encouraging nanoparticle concentrations for cellular development were $100 \mu \mathrm{g} / \mathrm{mL}$ of HAP and $2 \%$ SN. Subsequently, all the successive cellular examinations were operated using 100 $\mu \mathrm{g} / \mathrm{mL}$ of HAP and $2 \%$ SN. The GelMA-HAP-SN nanocomposite group presented the highest cellular viability on day 3 (91.87\%; Figure 2C). Cellular viability in the GelMAHAP and GelMA-SN hydrogels was $82.51 \%$ and $87.53 \%$, respectively. 
Additionally, cells encapsulated in GelMA-HAP-SN offered the most sizable cell-spreading area (Figure 2D), which was $3552.476 \pm 69.25 \mu^{2}$, compared with $2553.835 \pm 83.92 \mu \mathrm{m}^{2}$ of GelMA-SN and $2545.941 \pm$ $72.83 \mu \mathrm{m}^{2}$ of GelMA-HAP hydrogel samples.

\section{Osteogenic Activity of MSCs Embedded in HAP-SN Nanocomposite GelMA Hydrogel}

To determine the osteogenic activity of MSCs embedded in the hydrogel, the expression of osteogenic genes (ALP, RUNX2, OCN, and OPN) in the cells was first analyzed by RT-PCR at preset days 7 and 14. The highest gene expression level was found in the GelMA-HAP-SN nanocomposite hydrogel (Figure 3A-D). On day 7, the MSCs in GelMA-HAP-SN hydrogel demonstrated $\sim 25$-, $\sim 35-, \sim 16$ - and $\sim 13$-fold upregulated expression of ALP, RUNX2, OCN, and OPN compared with the blank GelMA group. Correspondingly, on day 14, GelMA-HAP-SN hydrogel revealed the highest expression of ALP, RUNX2, OCN, and OPN with 44-, 75-, 36- and 23fold elevation. The gene expression level of the GelMA-SN hydrogel group was increased, which was significantly lower than the GelMA-HAP-SN nanocomposite group.

We further evaluated osteogenesis-related protein expression levels (ALP, RUNX2, OCN, and OPN) of MSCs seeded and cultured in the hydrogel by immunostaining (Figure 3E). The quantification outcomes of the stained images were uniform with the above gene expression analysis, in which the MSCs in GelMA-HAP-SN scaffolds expressed the highest level of the four aforementioned proteins. Following a 14day culture, the proportionate fold increase in ALP, OCN, OPN, and RUNX2 protein was $\sim 27, \sim 45, \sim 20$, and $\sim 18$, respectively, compared with those of the GelMA hydrogel (Figure 3F-I).

\section{Matrix Mineralization Analysis of MSC Cultured with HAP and or SN Nanoparticles}

First, we cultured MSCs with $100 \mu \mathrm{g} / \mathrm{mL}$ HAP, $2 \% \mathrm{SN}$, or both in $2 \mathrm{D}$ culture. The staining was processed on days 14 and 28. The GelMA-HAP-SN group presented the most calcium nodule formation (Figure 4A). The quantification results on day 28 demonstrated a $\sim 1$-4-fold increase when the MSCs were cultured with HAP and SN simultaneously, which were higher than the other three groups.

The staining results of 3D culture samples are displayed in Figure 4B. Consistent with the results of the 2D culture, GelMA-HAP-SN presented the most intensified staining. Additionally, the coloration quantification showed that GelMA-HAP-SN promotes a $\sim 1.2$-fold and $\sim 1.8$-fold increase of mineralized extracellular matrix production on days 14 and 28, respectively, compared with the pure GelMA group (Figure 4C and D).

\section{In vivo Bone Regeneration Analysis}

We further explored the nanocomposite hydrogel's in vivo bone regeneration ability with a critical-sized calvaria defect model on the rat. The MSCs embedded in GelMA-HAP-SN nanocomposite hydrogel were injected into the defects and cross-linked in situ (as displayed in Figure 5A-B). All the rats were euthanized with anesthesia and an overdose of pentobarbital eight-week post-surgery for tissue collection. First, we used high-resolution micro-CT to appraise the bone regeneration rate. The $3 \mathrm{D}$ reconstruction demonstrated that the outstanding defect of the rats administered with the GelMA-HAP-SN nanocomposite hydrogel was reduced compared with the three remaining groups (GelMA, GelMAHAP, and GelMA-SN; Figure 5C). The quantification conclusions of bone regeneration are manifested in Figure 5D$G$ with bone mineral density (BMD), new bone volume (BV) and its ratio to tissue volume (BV/TV \%), and the bone surface/all surface analysis (BS/AS \%). The defects treated with GelMA-HAP-SN nanocomposite hydrogel, GelMASN, GelMA-HAP, and GelMA were repleted with newly formed bone at $29.26 \%, 43.82 \%, 42.37 \%$, and $61.73 \%$, as shown in the BS/AS \% chart, respectively. Additionally, the BV/TV of GelMA-HAP-SN nanocomposite hydrogel was exceptionally above that of the other three groups.

We further applied $\mathrm{H}$ and $\mathrm{E}$ and Goldner-Masson trichrome staining to the regenerated bone structure analysis. The defect treated with GelMA-HAP-SN nanocomposite hydrogel was $\sim 65 \%$ suffused with newly formed bone, which was the highest in the four groups (Figure 6A-B). Defects treated with GelMA-SN hydrogel were observed with lateral bone occupation, although there were gaps between the edges. The GelMA-HAP and GelMA groups regenerated a finite bone tissue on the edges, and the defects were mostly enclosed with soft tissue. Moreover, the GelMA-HAP-SN nanocomposite hydrogel vitalized the highest expression of new bone formation and vascularized tissue development (Figure 6C-D). Consistently, the Goldner-Masson staining (Figure 7) results proved that more new bone construction and osteoid matrix were established in the defect area when treated with the GelMA-HAP-SN nanocomposite hydrogel. Conversely, defects treated with the GelMA- 

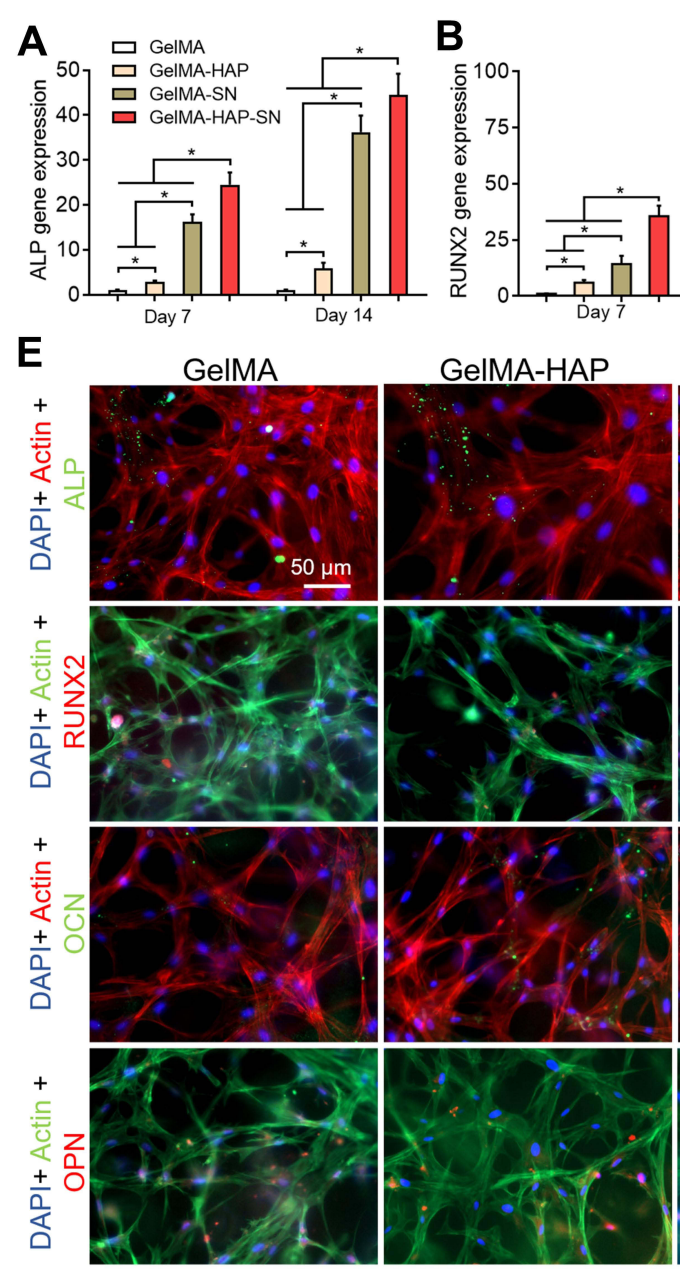

B

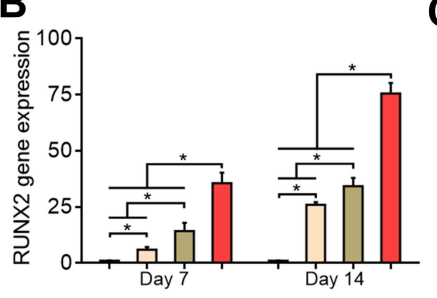

GeIMA-SN
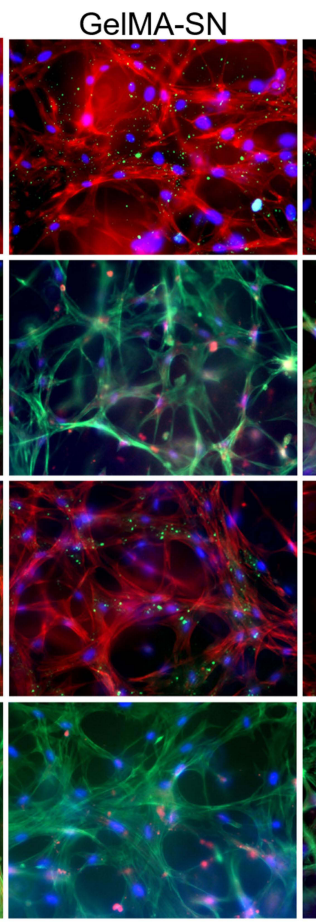

G

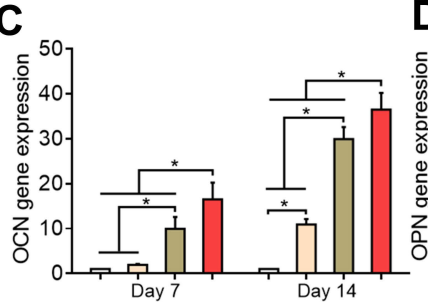

GelMA-HAP-SN
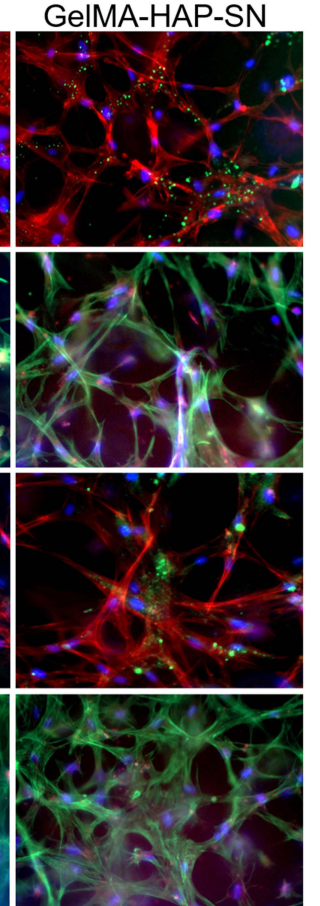

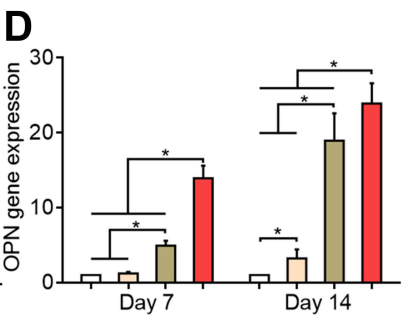

$\mathbf{F}$
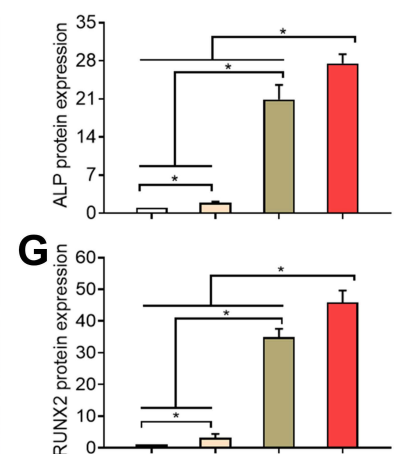

$\mathrm{H}$

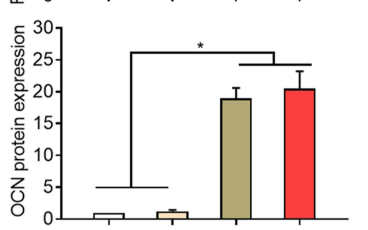

I

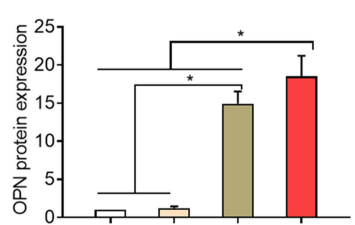

Figure 3 Osteogenesis-related biomarker expression. (A-D) Quantitative RT-PCR analysis of MSCs' ALP, RUNX2, OCN, and OPN gene expression when cultured with GeIMA, GelMA-HAP, GeIMA-SN, and GelMA-HAP-SN hydrogel on days 7 and I4. (E) Immunofluorescence staining of ALP (green), RUNX2 (red), OCN (green), and OPN (red) with DAPI-labeled nuclei (blue) and phalloidin-labeled actin (opposite to the proteins); (F-I) Quantification analysis of the immunofluorescence staining results indicating ALP, RUNX2, OCN, and OPN protein expression. $* P<0.05$ indicates statistical difference.

HAP hydrogel and GelMA hydrogel were primarily infused with fibrous connective tissue without credible bone-healing. Collectively, the GelMA-HAP-SN nanocomposite hydrogel caused the most effective bone regeneration in the defect area.

\section{Discussion}

Bone grafts have been widely used to promote bone regeneration, and their efficiency relies on their bioactivity and ability to boost the osteogenic activity. Bone tissue engineering hydrogels are a potentially ideal solution to treat bone defects due to their inherent capacity as a platform to integrate ECM, stem cells, and osteoinductive ingredients; thus, stimulating bone-healing efficiently. Besides, the hydrogel allows further modification to achieve bionic bone properties and could be injected into the bone defect area through a minimally invasive strategy. ${ }^{39-41}$ This study developed a biomimetic bone tissue engineering material with a quickly available strategy, in which nano HAP and SN were introduced into the MSCs encapsulated GelMA hydrogel. The injectable GelMA-HAP-SN hydrogel demonstrated excellent bone regeneration ability in vivo and in vitro.

The hydrogel injectability was comprehensively assessed regarding viscosity, injection force, and practical injection examination of various needle sizes. Additionally, the nanocomposite hydrogel was well injectable within the finest needle of $25 \mathrm{G}$, indicating that the invasive passage was minimized. The improved injectability attributed to shear thinning caused by SN simultaneously simplified the hydrogel's preparation process. Compared with the hours to days required for other studies, ${ }^{12,13}$ the hydrogel we designed took only seconds and can achieve similar or even better injectability. 


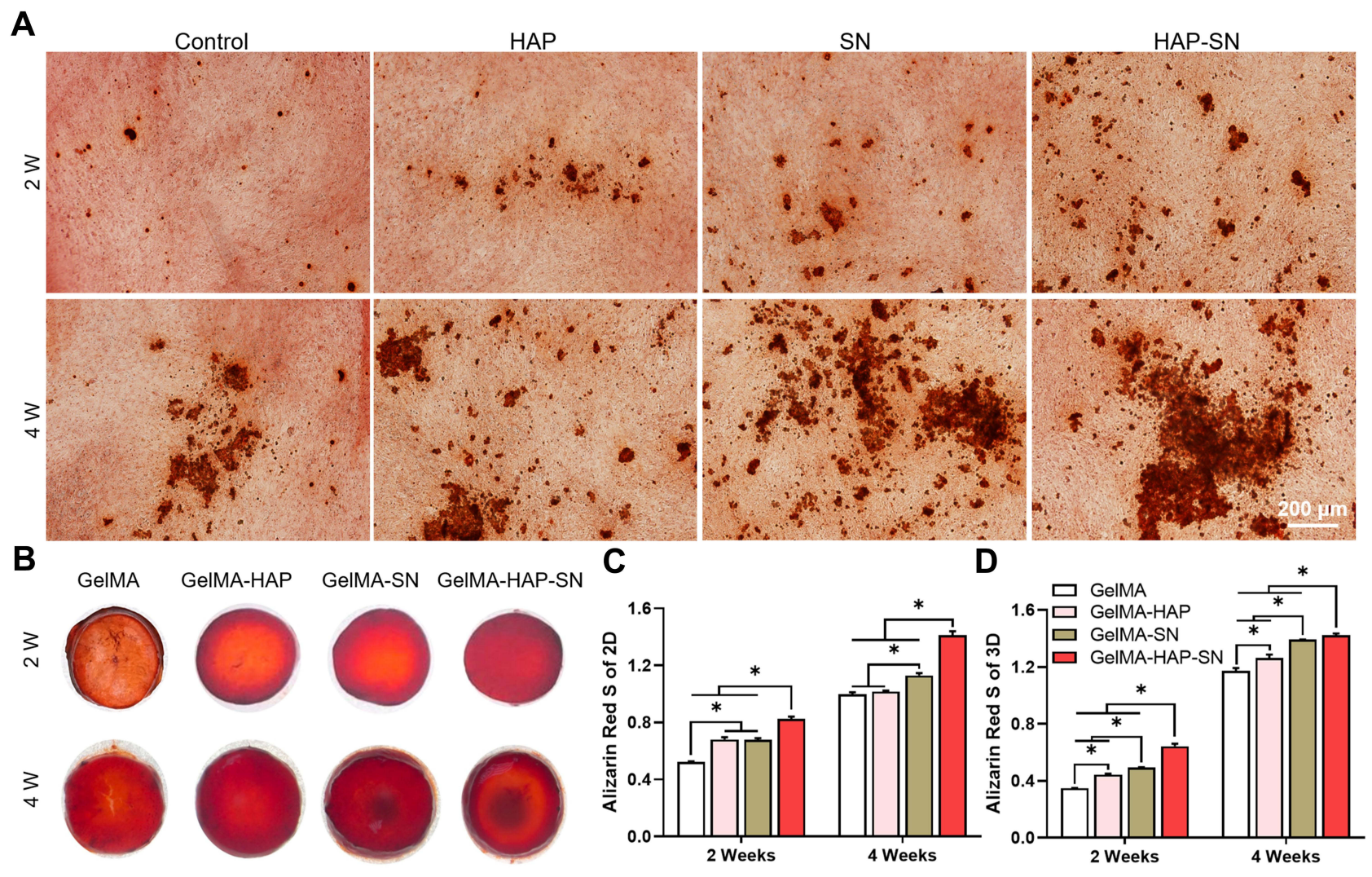

Figure 4 Matrix mineralization analysis. (A) Depictive images of Alizarin Red staining of the MSCs 2D-cultured with HAP, SN, and HAP + SN on days I4 and 28. (B) Exterior presence of Alizarin Red staining of the MSCs 3D-cultured with GelMA, GelMA-HAP, GelMA-SN, and GelMA-HAP-SN hydrogel on days I4 and 28. (C-D) Quantification analysis of the calcium deposits in nanoparticle-based-2D-cultured and GelMA-based-3D-cultured surroundings on days 14 and 28 , respectively. $* P<0.05$ indicates statistical difference.

Hydrogels with similar structure and composition to bone ECM have been proven beneficial for preserving the cellular bioactivity of encapsulated MSC cells. ${ }^{30,42}$ In this study, as the fundamental component of GelMA hydrogel is gelatin, which is collagen in a hydrolyzed form, introducing nano HAP into the GelMA hydrogel promoted the hydrogel's compositional similarities to the bone composition at the nanoscale. Besides, adding SN enhanced the compositional similarity as its degradation products, such as magnesium ions, are essential components in natural bone. ${ }^{43}$ Thus, the GelMA-HAP-SN hydrogel would be an excellent biomimetic cell-loading platform regarding compositional similarity.

The size and distribution of hydrogel pores are crucial for conducting cellular behavior, penetration of nutrients, and waste discharge. ${ }^{44-46}$ The hydrogels used in this study present similar pore size $(\sim 150 \mu \mathrm{m})$ to previous studies, which are suitable for cell ingrowth, cell migration, and cell-matrix interaction. ${ }^{30}$ Adding nano HAP and SN does not change the pore size because we believe the key variables determining pore sizes are the prepolymer concentration and UV radiation time for the GelMAHAP-SN hydrogel.

Hydrogel stiffness is also critical for cellular viability, proliferation, and differentiation. It is believed that hydrogel with $\sim 30 \mathrm{KPa}$ compression modulus is the optimized stiffness for encapsulated MSCs' osteogenic differentiation. ${ }^{30,47,48}$ Furthermore, adding HAP and SN enhanced the hydrogel stiffness (Figure 1H). Apart from the intrinsic mechanic strength of bioceramic particles, the increase in stiffness was also due to the interaction between the nanosilicates and the interaction between the nanosilicates and the hydrogel backbone. Thus, it means that the nanocomposite hydrogel required a shorter UVcross-linking time to reach the optimized stiffness of $\sim 30$ $\mathrm{KPa}$. Although UV radiation in a certain time range is considered safe for encapsulated cells, we believe a shorter UV exposure will benefit cellular preservation. This is because the encapsulated cells were usually fragile initially, as they were newly detached from the culture flask with trypsin; less UV exposure is better for ensuring the viability of the encapsulated cells. 
A

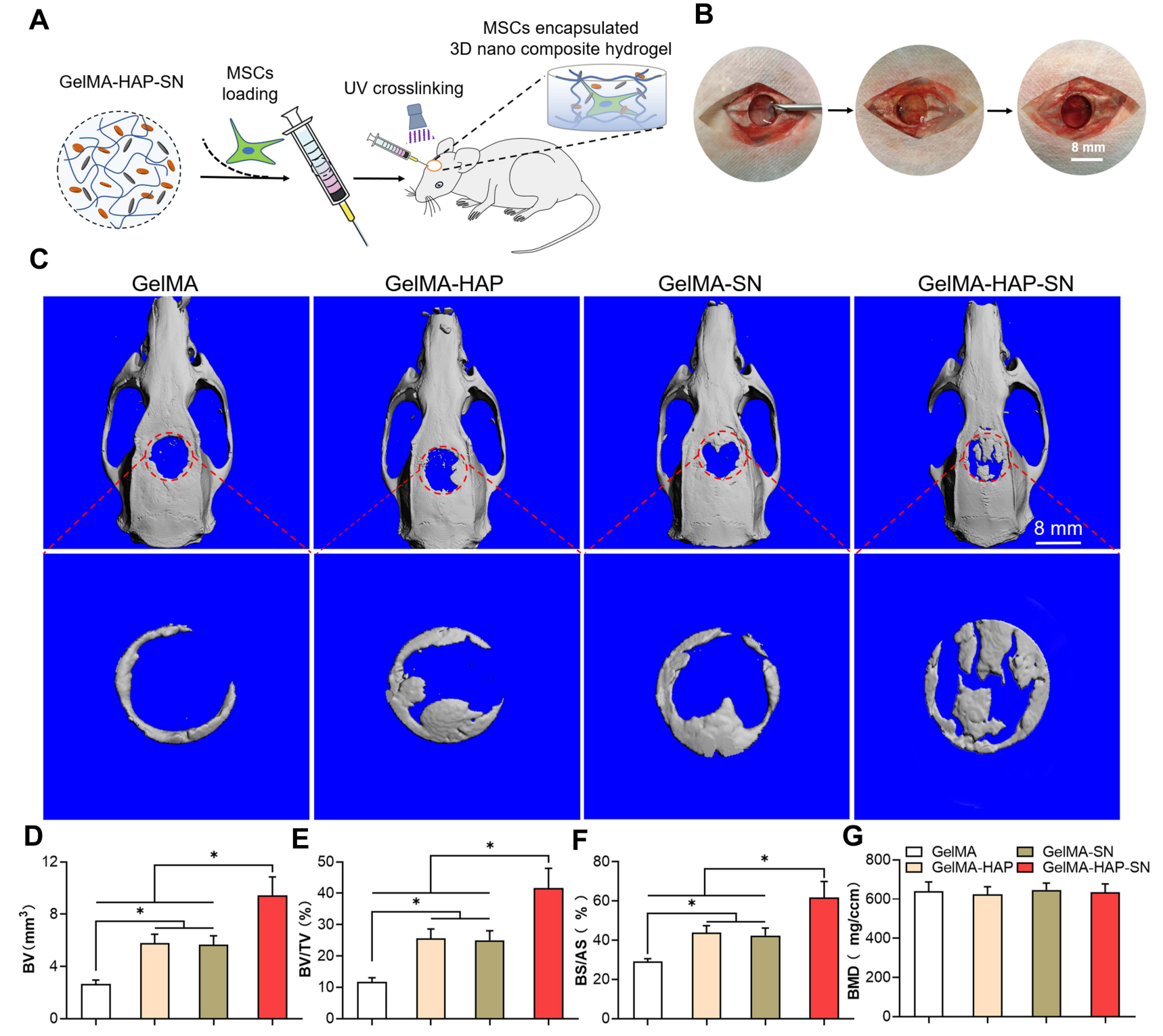

B

Figure 5 Formation of the rat calvaria defect model and in vivo bone regeneration assessment. (A-B) The process of constructing an 8-mm critical-size bone defect in rats' calvaria and the in situ UV-cross-linking of the GelMA-based MSCs embedded hydrogels. (C) Micro-CT scanning outcomes of the defective bone-healing in rats' calvaria treated with GeIMA, GelMA-HAP, GelMA-SN, and GelMA-HAP-SN hydrogel for eight weeks. The red circle indicates the initial area of the calvaria defect, which is also the area analyzed by micro-CT. (D-G) Quantification analysis of the in vivo-regenerated bone using BV, BV/TV, BS/AS, and BMD. *P < 0.05 indicates statistical difference.

The viability and spreading behavior of embedded MSCs were further analyzed to confirm the GelMA-HAPSN hydrogel's biocompatibility. All hydrogel groups presented excellent biocompatibility (Figure 2C). Similar to previous studies, ${ }^{30}$ more than $75 \%$ of the encapsulated cells were alive in all hydrogel groups on day 3. As mentioned before, the satisfied cell viability was mostly reliant on the compositional similarity and optimized compression modulus. The GelMA-HAP-SN hydrogel presented the highest cell viability on day 3, which was most probably due to the decreased UV exposure time in the beginning compared with other groups.
Additionally, MSCs grown on GelMA-HAP-SN hydrogel also exhibited a relatively higher proliferation rate and a larger cell-spreading area. More cells were found after day 3 in the nanocomposite hydrogel scaffold. It was reported that cell viability could be better maintained, and cell interaction could be enhanced at a higher cell density in 3D culture hydrogels. The cell-spreading area in the GelMA-HAP-SN hydrogel was $\sim 4.2$-fold bigger than that in the control group. The results agree with previous studies, in which SN could promote cellspreading due to its ability to stimulate cell adhesion, migration, and proliferation, as the $\mathrm{SN}$ could absorb 
A
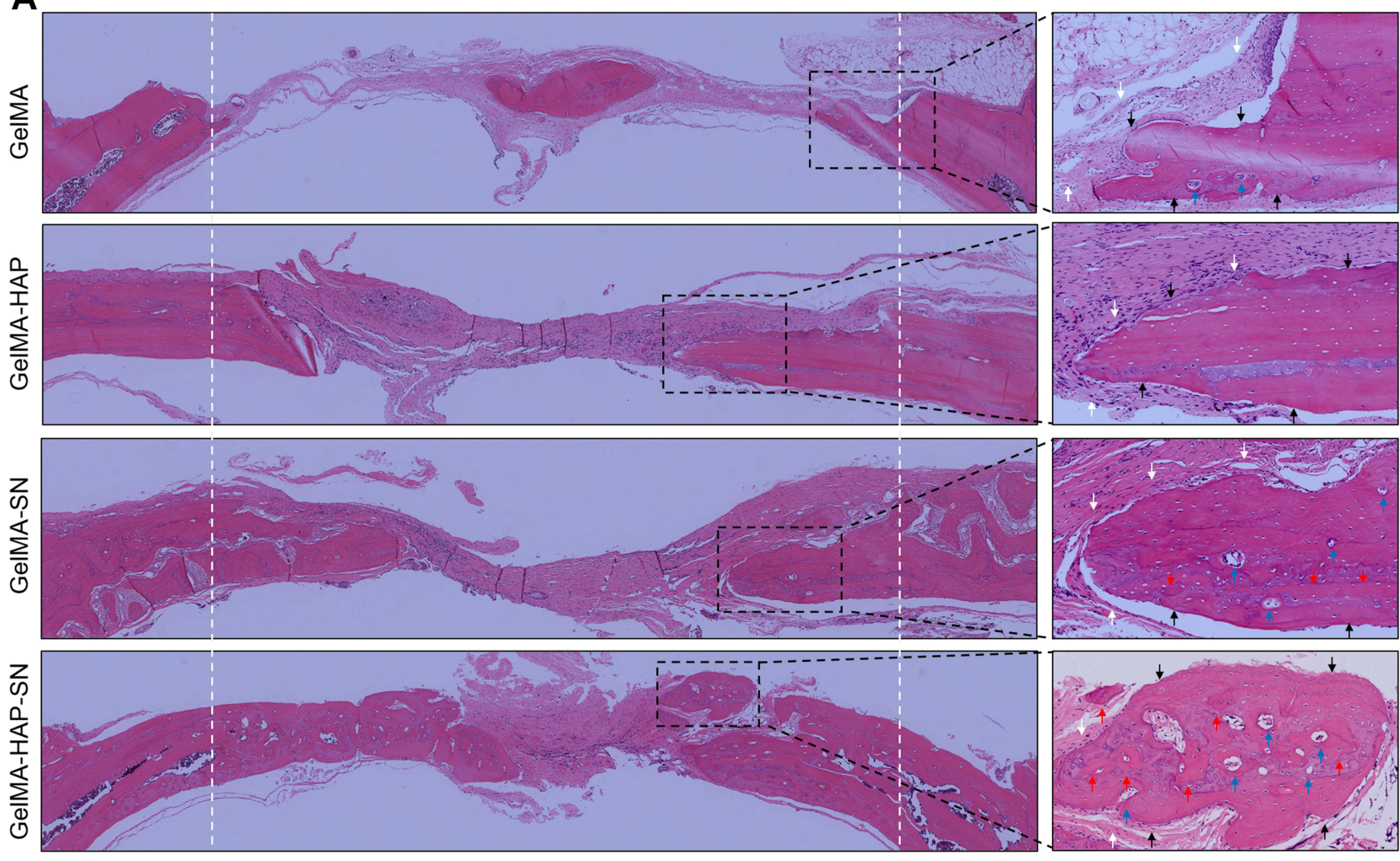

B

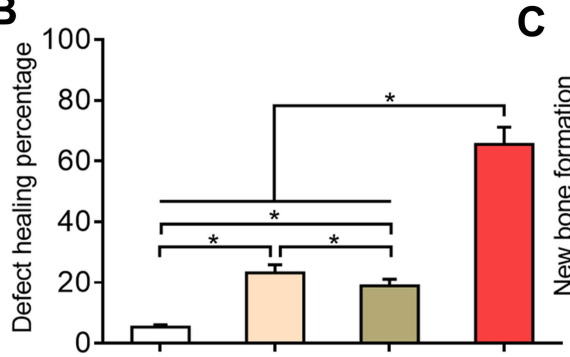

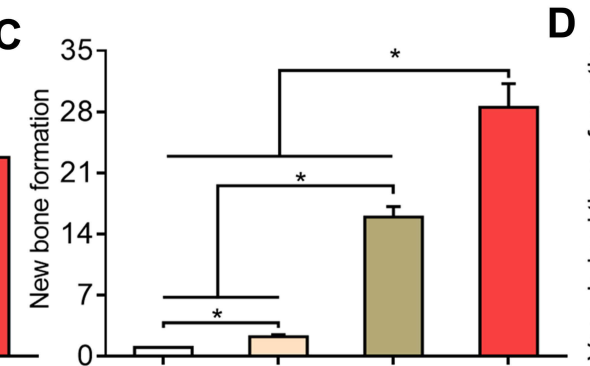

D

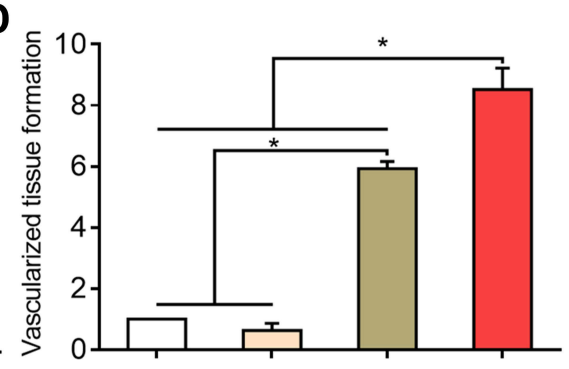

Figure $6 \mathrm{Hematoxylin}$ and eosin $(\mathrm{H}$ and $\mathrm{E})$ staining of the regenerated bone. $(\mathbf{A}) \mathrm{H}$ and $\mathrm{E}$ staining of the decalcified bone. White arrows indicate the fibrous tissue, and black arrows indicate the cortical bone. Red arrows indicate the osteoid, and blue arrows indicate neovascularization. (B) Healing percentage of the bone defects treated with GelMA, GelMA-HAP, GelMA-SN, and GelMA-HAP-SN hydrogel. (C-D) Quantification of new bone and vascularized tissue formation in the bone-healing interface from the defects treated with different GelMA-based hydrogels. $* P<0.05$ indicates statistical difference.

integrins and osteogenic proteins to its surface. ${ }^{28,49,50}$ It is believed that cells in more spread shapes are more prone to form cellular networks inside the hydrogels and develop multicellular aggregates.

The osteogenic activities of MSC cells embedded in different hydrogels were further recorded and analyzed (Figure 3). The highest osteogenic-related gene and protein expression levels of encapsulated MSCs were presented in the GelMA-HAP-SN hydrogel. As ALP expression is a typical event in the early osteogenesis process, ${ }^{6}$ the increased ALP activity in the two SNloaded hydrogels indicated that nanosilicates directly upregulated the osteogenic differentiation of MSCs from the beginning. Moreover, MSCs cultured with nanosilicates were characterized by an increase in RUNX2 activity, which is considered a critical transcription factor for stimulating several osteogenesis-related genes, such as collagen type I, OCN, and OPN. ${ }^{51}$ Concomitantly, significantly increased expression of OPN and OCN was detected in the GelMA-HAP-SN hydrogel. OCN is the most abundant non-collagenous protein and is usually regarded as an osteogenic maturation marker. ${ }^{52}$ OPN serves as a crucial factor during the bone remodeling process. ${ }^{53,54}$ Extracellular matrix production and mineralization of embedded MSCs were subsequently analyzed using Alizarin Red S staining. Consistent with previous 
A
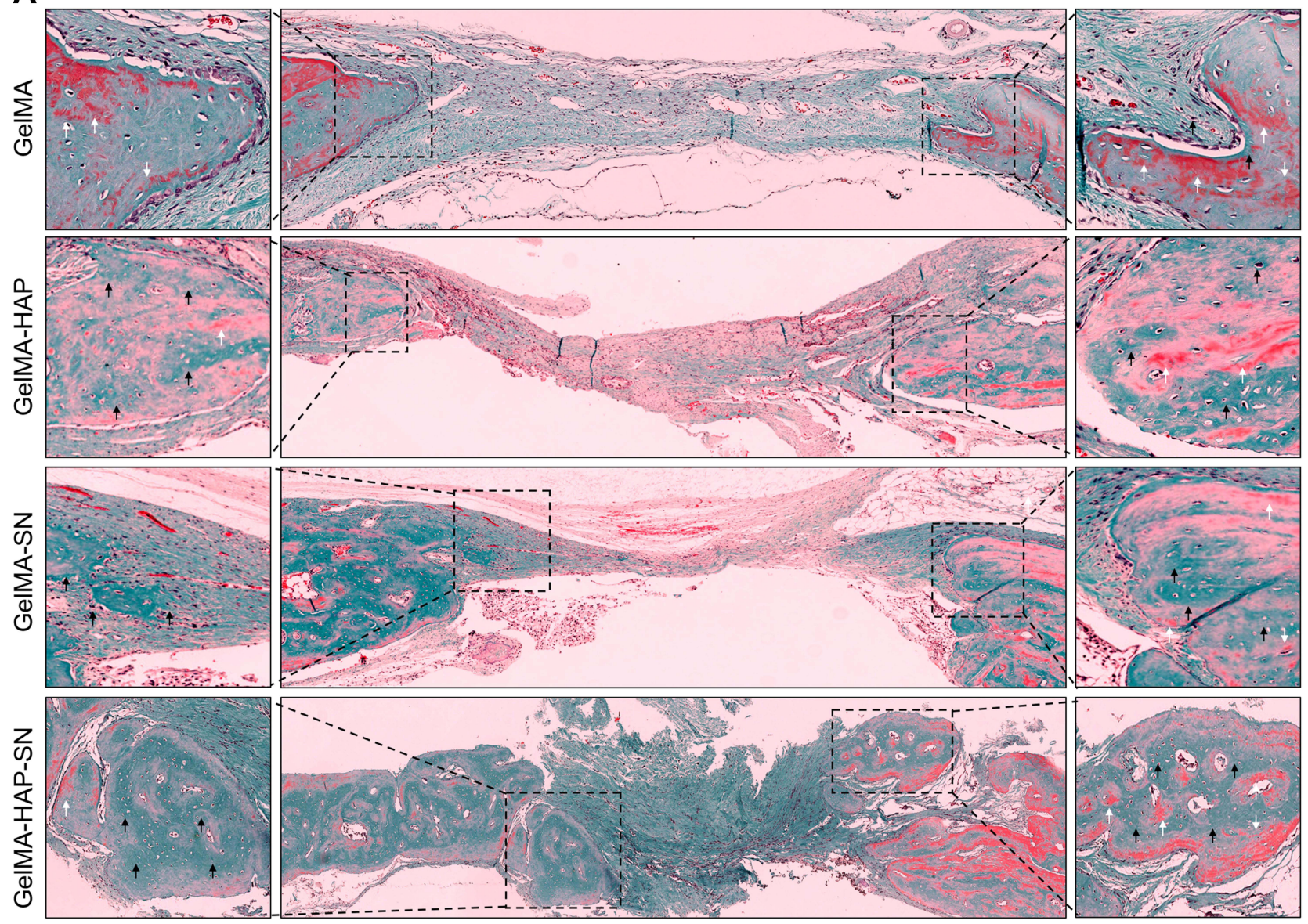

B

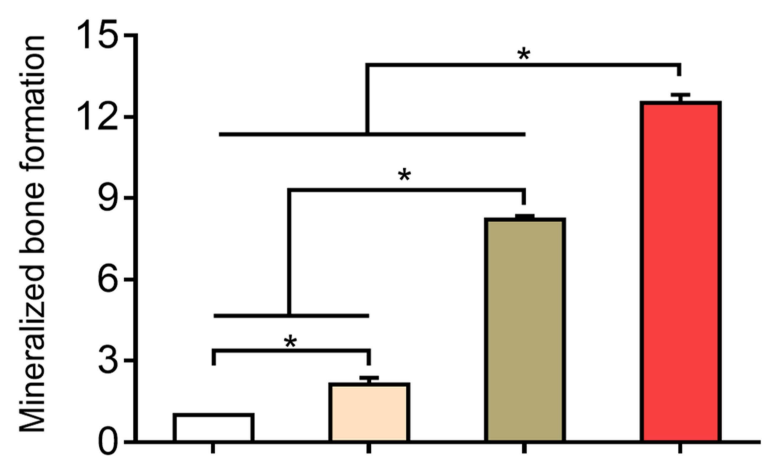

C

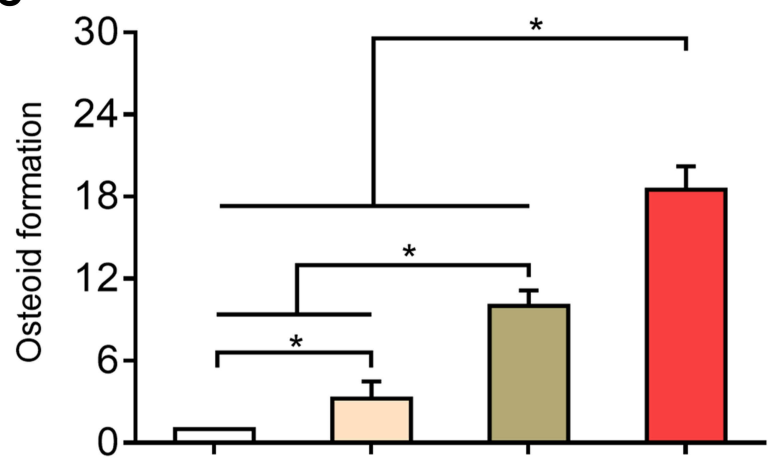

Figure 7 Goldner's trichrome staining of the regenerated bone. (A) Goldner's trichrome staining of newly regenerated bone. White arrows indicate the osteoid formation, and black arrows indicate the mineralized bone formation. (B-C) Quantification of mineralized bone and osteoid formation in the bone-healing interface from the defects treated with the various GelMA-based hydrogels. $* P<0.05$ indicates statistical difference.

studies, adding nanosilicate promoted calcium nodule formation in $2 \mathrm{D}$ and $3 \mathrm{D}$ culture conditions.

MSCs grown on the GelMA-HAP-SN hydrogel exhibited the most significant increase in osteogenic differentiation, protein expression, and subsequent matrix mannerization attributed to SN's bioactivity and the synergistic effect between nano HAP and nanosilicate to promote osteogenic differentiation. Apart from absorbing integrins and osteogenic proteins on its surface, ${ }^{28} \mathrm{SN}$ 's degradation products, orthosilicic acid $\left(\mathrm{Si}(\mathrm{OH})_{4}\right)$ and lithium, could directly upregulate osteogenic protein expression. ${ }^{32}$ The synergism between the two nanoparticles could be explained by $\mathrm{PO}_{4}{ }^{3-}$ and $\mathrm{Mg}^{2+}$ ions released from the HAP and $\mathrm{SN}$, respectively. It is believed that 
certain amounts of $\mathrm{PO}_{4}{ }^{3-}$ and $\mathrm{Mg}^{2+}$ ions could work coordinately to upregulate the osteogenic activity of MSC cells. ${ }^{30}$

To further validate the bone regeneration and healing ability of the GelMA-HAP-SN hydrogel in vivo, MSCencapsulated hydrogels were injected into the critical-sized cranial defect area in rats. The hydrogel's injectability and in situ cross-linking property offered the convenience of filling the irregular-shape bone defect we often meet in surgery. Thus, rather than trimming the bone edges to meet a specified shape of the commercial graft material, this GelMA-HAP-SN hydrogel can maximally preserve the local BV. Consistent with the above in vitro results, micro$\mathrm{CT}$ results revealed that the highest bone regeneration volume was presented in the GelMA-HAP-SN-treated rats eight weeks after transplantation. Histological analysis further confirmed GelMA-HAP-SN hydrogel's premium capacity to regenerate bone tissue. Besides, the new bone substituted with the GelMA-HAP-SN hydrogel possessed more new bone and vascular tissue formation (Figure 6). Thus, GelMA-HAP-SN hydrogel stimulates bone regeneration quantitatively and qualitatively. Altogether, the in vivo results reinforced the hypothesis that GelMAHAP-SN hydrogel can act as efficient biomimetic and osteogenic tissue engineering materials to enhance bone regeneration.

The mechanical properties of the regenerated bone tissue were not analyzed in this study because the calvaria defect area was not strictly strength demanding as it is not load-bearing. However, the newly formed bone can replace the hydrogel and may not possess satisfactory mechanical properties, limiting the hydrogels' use in loadbearing defects. Thus, a segmental bone defect model on larger animals may be needed in future studies. Also, longterm observation of bone-healing is necessary to determine the long-term outcome of using GelMA-HAP-SN hydrogels. Therefore, further biochemical cues, such as essential growth factors presented in the natural bone-healing process, could be introduced into the GelMA-HAP-SN hydrogel to ensure the regenerated bone tissue remodels well into natural bone.

These MSC-loaded GelMA-HAP-SN hydrogels are potentially useful graft materials to treat bone defects due to their integration of compositional similarity to natural bone, osteogenic cells, and osteoinductive factors. Additionally, porous biomimetic matrix possesses the potential to support and induce the growth of nerve and blood vessels, ${ }^{55}$ which is achievable by planting other seeds, such as endothelial progenitor cells (EPCs). ${ }^{56}$ The penetration of new blood vessels and nerve tissues into implant materials also plays a critical role in bone and other tissue regeneration. They can supply the necessary oxygen, nutrients, essential growth factors, neurofeedback, and neuro control. ${ }^{55}$ Specifically, GelMA has been widely used in tissue engineering with confirmed excellent biosafety. ${ }^{57}$ HAP is the most abundant mineral in natural human bone and has also been widely used as a part of bone graft materials. The biocompatibility of SN with a certain concentration is also proven in previous studies. $^{58}$ In our study, the biosafety of the injectable system verified the excellent bone regeneration capacity with the in vivo results. Thus, the biomimetic, biocompatible, bioactive, and injectable cell-loaded nanocomposite hydrogel holds great potential for advanced tissue and even organ regeneration.

\section{Conclusion}

This study designed an injectable MSC-encapsulated biomimetic nanoparticle co-loaded hydrogel system used for bone tissue regeneration. HAP in GelMA resulted in similarity to natural bone structure and ingredients, while loaded SN provided ideal injectability and osteoinductivity without exogenous growth factor. The nanoparticle coloaded system combined and amplified both advantages synergistically, explaining its more excellent injectability, cell activity, and osteogenesis in vitro and in vivo than simply HAP- or SN-loaded system. This study will contribute to the following studies for developing injectable biomimetic bone graft materials for treating bone defects.

\section{Acknowledgments}

This study was funded by the National Science Foundation of China (81802183 and 81871757), Medical Scientific Research Foundation of Guangdong Province (B2018078), President Foundation of Nanfang Hospital, Southern Medical University (2017C038 and 2017B030), and Outstanding Youths Development Scheme of Nanfang Hospital, Southern Medical University (2018J011).

\section{Disclosure}

The authors report no conflicts of interest in this work.

\section{References}

1. Mauffrey C, Barlow BT, Smith W. Management of segmental bone defects. J Am Acad Orthop Surg. 2015;23(3):143-153. 
2. Mauffrey C, Barlow BT, Smith W. Management of segmental bone defects. JAAOS. 2015;23(3):143-153.

3. Sakkas A, Wilde F, Heufelder M, Winter K, Schramm A. Autogenous bone grafts in oral implantology — is it still a "gold standard"? A consecutive review of 279 patients with 456 clinical procedures. Int $j$ Implant Dentistry. 2017;3(1):23. doi:10.1186/s40729-017-0084-4

4. Rogers GF, Greene AK. Autogenous bone graft: basic science and clinical implications. J Craniofacial Surg. 2012;23(1):323-327. doi:10.1097/SCS.0b013e318241dcba

5. Wang W, Yeung KW. Bone grafts and biomaterials substitutes for bone defect repair: a review. Bioactive Materials. 2017;2(4):224-247.

6. Sudhakar K, Mohanty R, Singh V. Evaluation of donor site morbidity associated with iliac crest bone harvest in oral and maxillofacial, reconstructive surgery. J Clin Diagnostic Res. 2017;11(6):ZC28.

7. Fernandez de Grado G, Keller L, Idoux-Gillet Y, et al. Bone substitutes: a review of their characteristics, clinical use, and perspectives for large bone defects management. $J$ Tissue Eng. 2018;9:2041731418776819. doi:10.1177/2041731418776819

8. Matassi F, Botti A, Sirleo L, Carulli C, Innocenti M. Porous metal for orthopedics implants. Clin Cases Mineral Bone Metab. 2013;10 (2): 111.

9. Saini M, Singh Y, Arora P, Arora V, Jain K. Implant biomaterials: a comprehensive review. World J Clin Cases. 2015;3(1):52. doi:10.12998/wjcc.v3.i1.52

10. Huang KQ, Liu GT, Gu ZP, Wu J. Tofu as excellent scaffolds for potential bone regeneration. Chinese Chem Lett. 2020;31 (12):3190-3194. doi:10.1016/j.cclet.2020.07.002

11. El-Sherbiny IM, Yacoub MH. Hydrogel scaffolds for tissue engineering: progress and challenges. Glob Cardiol Sci Pract. 2013;2013 (3):316-342.

12. Cui ZK, Kim S, Baljon JJ, Wu BM, Aghaloo T, Lee M. Microporous methacrylated glycol chitosan-montmorillonite nanocomposite hydrogel for bone tissue engineering. Nat Commun. 2019;10 (1):3523. doi:10.1038/s41467-019-11511-3

13. Wasupalli GK, Verma D. Injectable and thermosensitive nanofibrous hydrogel for bone tissue engineering. Mater Sci Eng C Mater Biol Appl. 2020;107:110343. doi:10.1016/j.msec.2019.110343

14. Xavier JR, Thakur T, Desai P, et al. Bioactive nanoengineered hydrogels for bone tissue engineering: a growth-factor-free approach. $A C S$ Nano. 2015;9(3):3109-3118. doi:10.1021/nn507488s

15. Vo T, Shah S, Lu S, et al. Injectable dual-gelling cell-laden composite hydrogels for bone tissue engineering. Biomaterials. 2016;83:1-11. doi:10.1016/j.biomaterials.2015.12.026

16. Xie X, Hu K, Fang D, Shang L, Tran SD, Cerruti M. Graphene and hydroxyapatite self-assemble into homogeneous, free standing nanocomposite hydrogels for bone tissue engineering. Nanoscale. 2015;7 (17):7992-8002. doi:10.1039/C5NR01107H

17. Vo TN, Ekenseair AK, Spicer PP, et al. In vitro and in vivo evaluation of self-mineralization and biocompatibility of injectable, dual-gelling hydrogels for bone tissue engineering. $J$ Controlled Release. 2015;205:25-34. doi:10.1016/j.jconrel.2014.11.028

18. Kim HD, Lee EA, An Y-H, et al. Chondroitin sulfate-based biomineralizing surface Hydrogels for bone tissue engineering. ACS Appl Mater Interfaces. 2017;9(26):21639-21650. doi:10.1021/acsami.7b04114

19. Mihaila SM, Reis RL, Marques AP, Gomes ME. Hydrogels in bone tissue engineering: a multi-parametric approach. In: $G E L S$ HANDBOOK: Fundamentals, Properties and Applications Volume 2: Applications of Hydrogels in Regenerative Medicine. World Scientific; 2016:165-197.

20. Kondiah PJ, Choonara YE, Kondiah PP, et al. A review of injectable polymeric hydrogel systems for application in bone tissue engineering. Molecules. 2016;21(11):1580. doi:10.3390/molecules21111580

21. De Witte T-M, Fratila-Apachitei LE, Zadpoor AA, Peppas NA. Bone tissue engineering via growth factor delivery: from scaffolds to complex matrices. Regenerative Biomaterials. 2018;5(4):197-211. doi:10.1093/rb/rby013
22. Nichol JW, Koshy ST, Bae H, Hwang CM, Yamanlar S, Khademhosseini A. Cell-laden microengineered gelatin methacrylate hydrogels. Biomaterials. 2010;31(21):5536-5544. doi:10.1016/j. biomaterials.2010.03.064

23. Yue K, Trujillo-de Santiago G, Alvarez MM, Tamayol A, Annabi N, Khademhosseini A. Synthesis, properties, and biomedical applications of gelatin methacryloyl (GelMA) hydrogels. Biomaterials. 2015;73:254-271. doi:10.1016/j.biomaterials.2015.08.045

24. Bao ZT, Gu ZP, Xu JB, Zhao M, Liu GT, Wu J. Acid-responsive composite hydrogel platform with space-controllable stiffness and calcium supply for enhanced bone regeneration. Chem Eng $J$. 2020;2:396.

25. Sadat-Shojai M, Khorasani M-T, Jamshidi A. 3-Dimensional cell-laden nano-hydroxyapatite/protein hydrogels for bone regeneration applications. Materials Sci Eng. 2015;49:835-843. doi:10.1016/ j.msec.2015.01.067

26. Ren B, Chen X, Du S, et al. Injectable polysaccharide hydrogel embedded with hydroxyapatite and calcium carbonate for drug delivery and bone tissue engineering. Int $J$ Biol Macromol. 2018;118:1257-1266. doi:10.1016/j.ijbiomac.2018.06.200

27. Ran J, Jiang P, Liu S, et al. Constructing multi-component organic/ inorganic composite bacterial cellulose-gelatin/hydroxyapatite double-network scaffold platform for stem cell-mediated bone tissue engineering. Materials Sci Eng. 2017;78:130-140. doi:10.1016/j. msec.2017.04.062

28. Gaharwar AK, Cross LM, Peak CW, et al. 2D Nanoclay for Biomedical Applications: regenerative Medicine, Therapeutic Delivery, and Additive Manufacturing. Adv Mater. 2019;31 (23):1900332. doi:10.1002/adma.201900332

29. Cheng H, Yue K, Kazemzadeh-Narbat M, et al. Mussel-inspired multifunctional hydrogel coating for prevention of infections and enhanced osteogenesis. ACS Appl Mater Interfaces. 2017;9 (13):11428-11439. doi:10.1021/acsami.6b16779

30. Cheng H, Chabok R, Guan X, et al. Synergistic interplay between the two major bone minerals, hydroxyapatite and whitlockite nanoparticles, for osteogenic differentiation of mesenchymal stem cells. Acta biomaterialia. 2018;69:342-351. doi:10.1016/j.actbio.2018.01.016

31. Zhu Z, Yin J, Guan J, et al. Lithium stimulates human bone marrow derived mesenchymal stem cell proliferation through GSK-3 $\beta$-dependent $\beta$-catenin/Wnt pathway activation. FEBS J. 2014;281 (23):5371-5389. doi:10.1111/febs.13081

32. Zhou X, Moussa FM, Mankoci S, et al. Orthosilicic acid, Si (OH) 4, stimulates osteoblast differentiation in vitro by upregulating miR$146 \mathrm{a}$ to antagonize NF- $\mathrm{KB}$ activation. Acta biomaterialia. 2016;39:192-202. doi:10.1016/j.actbio.2016.05.007

33. Reffitt D, Ogston N, Jugdaohsingh R, et al. Orthosilicic acid stimulates collagen type 1 synthesis and osteoblastic differentiation in human osteoblast-like cells in vitro. Bone. 2003;32(2):127-135. doi:10.1016/S8756-3282(02)00950-X

34. Vimalraj S, Arumugam B, Miranda P, Selvamurugan N. Runx2: structure, function, and phosphorylation in osteoblast differentiation. Int $J$ Biol Macromol. 2015;78:202-208. doi:10.1016/j. ijbiomac.2015.04.008

35. Pérez-Campo FM, Santurtún A, García-Ibarbia C, et al. Osterix and RUNX2 are transcriptional regulators of sclerostin in human bone. Calcif Tissue Int. 2016;99(3):302-309. doi:10.1007/s00223-0160144-4

36. Nadernezhad A, Caliskan OS, Topuz F, Afghah F, Erman B, Koc B. Nanocomposite Bioinks Based on Agarose and 2D Nanosilicates with Tunable Flow Properties and Bioactivity for 3D Bioprinting. ACS Applied Bio Materials. 2019;2(2):796-806. doi:10.1021/ acsabm. 8 b00665

37. Cidonio G, Cooke M, Glinka M, Dawson J, Grover L, Oreffo R. Printing bone in a gel: using nanocomposite bioink to print functionalised bone scaffolds. Materials Today Bio. 2019;4:100028. doi:10.1016/j.mtbio.2019.100028 
38. González-Gil AB, Lamo-Espinosa JM, Muiños-López E, et al Periosteum-derived mesenchymal progenitor cells in engineered implants promote fracture healing in a critical-size defect rat model. $J$ Tissue Eng Regen Med. 2019;13(5):742-752. doi:10.1002/ term. 2821

39. Bendtsen ST, Wei M. Synthesis and characterization of a novel injectable alginate-collagen-hydroxyapatite hydrogel for bone tissue regeneration. $J$ Materials Chem B. 2015;3(15):3081-3090. doi:10.1039/C5TB00072F

40. Saekhor K, Udomsinprasert W, Honsawek S, Tachaboonyakiat W. Preparation of an injectable modified chitosan-based hydrogel approaching for bone tissue engineering. Int J Biol Macromol. 2019;123:167-173. doi:10.1016/j.ijbiomac.2018.11.041

41. Hou S, Wang X, Park S, Jin X, Ma PX. Rapid self-integrating, injectable hydrogel for tissue complex regeneration. Adv Healthcare Mater. 2015;4(10):1491-1495. doi:10.1002/adhm.201500093

42. Tsou Y-H, Khoneisser J, Huang P, Xu X. Hydrogel as a bioactive material to regulate stem cell fate. Bioactive Materials. 2016;1 (1):39-55. doi:10.1016/j.bioactmat.2016.05.001

43. Von Euw S, Wang Y, Laurent G, et al. Bone mineral: new insights into its chemical composition. Sci Rep. 2019;9:254.

44. Perez RA, Mestres G. Role of pore size and morphology in musculo-skeletal tissue regeneration. Materials Sci Eng. 2016;61:922-939. doi:10.1016/j.msec.2015.12.087

45. Brandl F, Sommer F, Goepferich A. Rational design of hydrogels for tissue engineering: impact of physical factors on cell behavior Biomaterials. 2007;28(2):134-146. doi:10.1016/j.biomateria 1s.2006.09.017

46. Fan C, Wang D-A. Macroporous hydrogel scaffolds for three-dimensional cell culture and tissue engineering. Tissue Eng Part B Rev. 2017;23(5):451-461. doi:10.1089/ten.teb.2016.0465

47. Lv H, Wang H, Zhang Z, et al. Biomaterial stiffness determines stem cell fate. Life Sci. 2017;178:42-48. doi:10.1016/j.1fs.2017.04.014

48. Kim TH, An DB, Oh SH, Kang MK, Song HH, Lee JH. Creating stiffness gradient polyvinyl alcohol hydrogel using a simple gradual freezing-thawing method to investigate stem cell differentiation behaviors. Biomaterials. 2015;40:51-60. doi:10.1016/j.biomater ials.2014.11.017
49. Gaharwar AK, Schexnailder PJ, Kline BP, Schmidt G. Assessment of using Laponite ${ }^{\circledR}$ cross-linked poly (ethylene oxide) for controlled cell adhesion and mineralization. Acta biomaterialia. 2011;7(2):568-577. doi:10.1016/j.actbio.2010.09.015

50. Topuz F, Nadernezhad A, Caliskan OS, Menceloglu YZ, Koc B. Nanosilicate embedded agarose hydrogels with improved bioactivity. Carbohydr Polym. 2018;201:105-112. doi:10.1016/j. carbpol.2018.08.032

51. Bruderer M, Richards R, Alini M, Stoddart MJ. Role and regulation of RUNX2 in osteogenesis. Eur Cell Mater. 2014;28(28):269-286. doi:10.22203/eCM.v028a19

52. Tsao Y-T, Huang Y-J, Wu -H-H, Liu Y-A, Liu Y, Lee O. Osteocalcin mediates biomineralization during osteogenic maturation in human mesenchymal stromal cells. Int $J$ Mol Sci. 2017;18(1):159. doi:10.3390/ijms18010159

53. Singh A, Gill G, Kaur H, Amhmed M, Jakhu H. Role of osteopontin in bone remodeling and orthodontic tooth movement: a review. Prog Orthod. 2018;19(1):1-8. doi:10.1186/s40510-018-0216-2

54. Terai K, Takano-Yamamoto T, Ohba Y, et al. Role of osteopontin in bone remodeling caused by mechanical stress. J Bone Mineral Res. 1999;14(6):839-849. doi:10.1359/jbmr.1999.14.6.839

55. Marrella A, Lee TY, Lee DH, et al. Engineering vascularized and innervated bone biomaterials for improved skeletal tissue regeneration. Materials Today. 2018;21(4):362-376.

56. Peng J, Chen L, Peng K, et al. Bone Marrow Mesenchymal Stem Cells and Endothelial Progenitor Cells Co-Culture Enhances Large Segment Bone Defect Repair. J Biomed Nanotechnol. 2019;15 (4):742-755. doi:10.1166/jbn.2019.2735

57. Yue K, Trujillo-de Santiago G, Alvarez MM, Tamayol A, Annabi N, Khademhosseini A. Synthesis, properties, and biomedical applications of gelatin methacryloyl (GelMA) hydrogels. Biomaterials. 2015;73:254-271.

58. Gaharwar AK, Mihaila SM, Swami A, et al. Bioactive silicate nanoplatelets for osteogenic differentiation of human mesenchymal stem cells. Adv Mater. 2013;25(24):3329-3336.
International Journal of Nanomedicine

\section{Publish your work in this journal}

The International Journal of Nanomedicine is an international, peerreviewed journal focusing on the application of nanotechnology in diagnostics, therapeutics, and drug delivery systems throughout the biomedical field. This journal is indexed on PubMed Central, MedLine, CAS, SciSearch ${ }^{\mathbb{B}}$, Current Contents ${ }^{\mathbb{B}} /$ Clinical Medicine, $^{2}$
Journal Citation Reports/Science Edition, EMBase, Scopus and the Elsevier Bibliographic databases. The manuscript management system is completely online and includes a very quick and fair peer-review system, which is all easy to use. Visit http://www.dovepress.com/ testimonials.php to read real quotes from published authors. 